\title{
REASSURANCE-SEEKING BEHAVIOUR, INTOLERANCE OF UNCERTAINTY, AND NEUROCOGNITIVE FUNCTIONING IN OBSESSIVE-COMPULSIVE DISORDER by \\ Erin Alexandra Orr
}

Bachelor of Science, St Francis Xavier University, 2015

\author{
A thesis \\ presented to Ryerson University \\ in partial fulfillment of the requirements \\ for the degree of \\ Master of Arts \\ in the Program of \\ Psychology
}

Toronto, Ontario, Canada, 2017

(C) Erin Orr, 2017 


\section{AUTHOR'S DECLARATION}

I hereby declare that I am the sole author of this thesis. This is a true copy of the thesis, including any required final revisions, as accepted by my examiners.

I authorize Ryerson University to lend this thesis to other institutions or individuals for the purpose of scholarly research.

I further authorize Ryerson University to reproduce this thesis by photocopying or by other means, in total or in part, at the request of other institutions or individuals for the purpose of scholarly research.

I understand that my thesis may be made electronically available to the public. 
Reassurance-Seeking Behaviour, Intolerance of Uncertainty, and Neurocognitive Functioning in Obsessive-Compulsive Disorder

Erin Orr

Master of Arts, 2017

Clinical Psychology

Ryerson University

\begin{abstract}
Obsessive-compulsive disorder (OCD) is a psychological condition involving excessive reassurance-seeking (ERS), or repeated questioning of others about something perceived as threatening; low cognitive confidence; and intolerance of uncertainty (IU; Cougle et al., 2012; Nedeljkovic et al., 2009; Parrish, 2009). Relationships between these symptoms and several domains of cognitive functioning were examined in the present study. Thirty-five OCD patients and 34 healthy controls completed a battery of psychological questionnaires and neurocognitive measures. Results indicated that OCD patients reported greater levels of ERS than healthy controls in three different situations. Furthermore, significant relationships were identified between: (1) decision-related ERS and decision-making impairment, (2) cognitive confidence and decision-related ERS, and (3) a particular form of IU, inhibitory IU, and decision-making impairment. These findings are important, as these psychological symptoms are associated with negative outcomes. The relationships identified provide an important foundation for future research and the development of targeted interventions for these symptoms.
\end{abstract}




\section{ACKNOWLEDGEMENTS}

I would first like to thank my thesis supervisor Dr. Tisha Ornstein of the Department of Psychology at Ryerson University. Dr. Ornstein was always available for consultation and she offered me support and guidance over the past two years. Dr. Ornstein also took the time to edit my work carefully and provide me with helpful feedback, while also encouraging me to make this work my own.

I would also like to thank Dr. Neil Rector and his research team at Sunnybrook Health Sciences Centre, who made this work possible through their openness to collaboration.

Furthermore, I would like to acknowledge my second committee member Dr. Kristin Vickers of the Psychology Department at Ryerson University. I am so grateful for her very valuable comments on this thesis.

Lastly, I would like to thank Ceilagh MacDonald and Natalie Slavat for helping with data collection, scoring, and entry for this project. I am so appreciative of their willingness to dedicate time to these endeavors.

Erin Orr 


\section{DEDICATION}

This thesis is dedicated to my wonderful parents and brother, and to my thoughtful and caring partner, Gerard O’Leary. Thank you all so much for your support throughout my Master's degree. I would also like to thank my M.A. cohort for their friendship, support, and encouragement over the past two years. Finally, thank you to my supervisor Dr. Ornstein and to the other members of the Cognitive Neuroscience Lab for their continued support and advisement throughout this process. 


\section{TABLE OF CONTENTS}

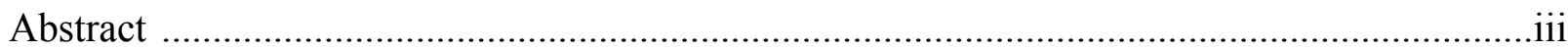

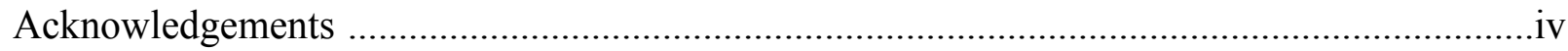

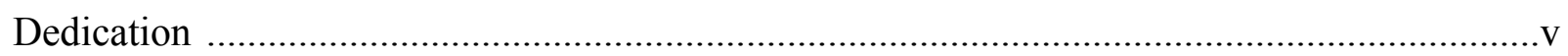

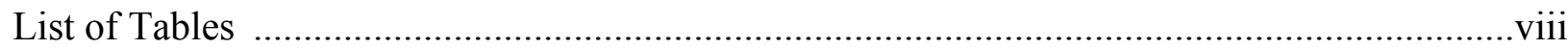

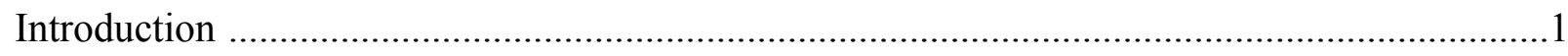

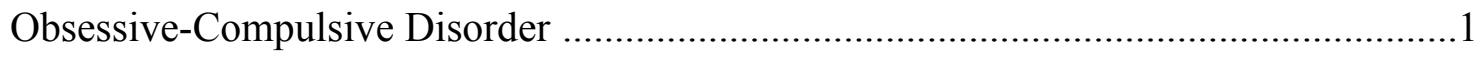

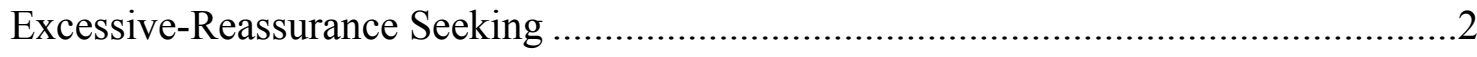

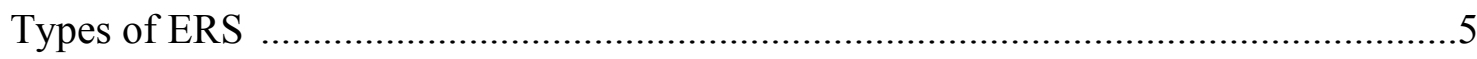

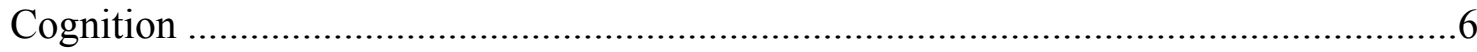

Decision-Making and Indecisiveness ......................................................................

ERS and Cognitive Confidence ……………….....................................................12

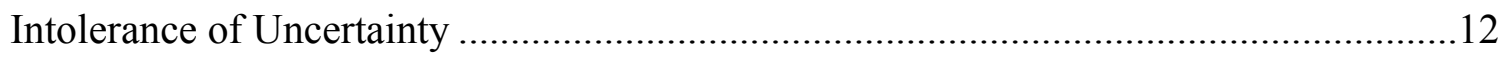

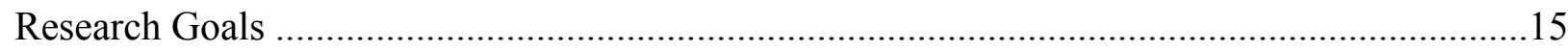

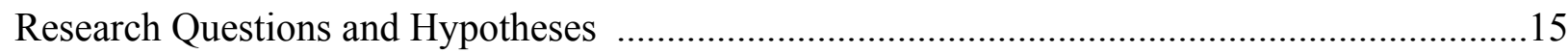

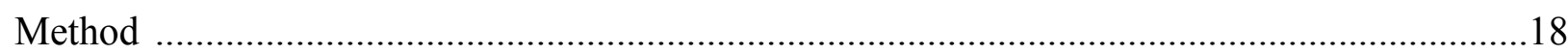

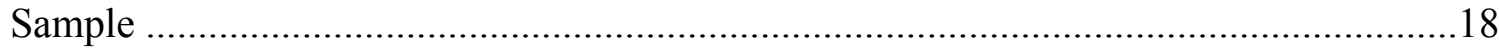

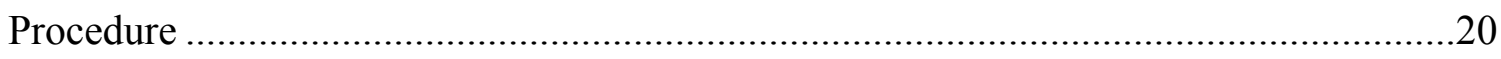

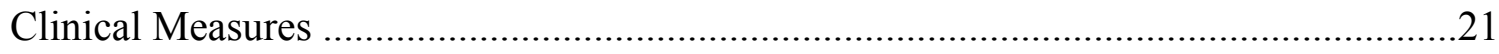

Screening Measures for Healthy Controls …………..................................................22

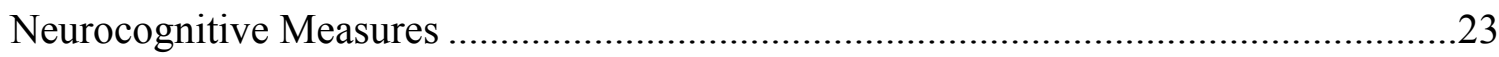

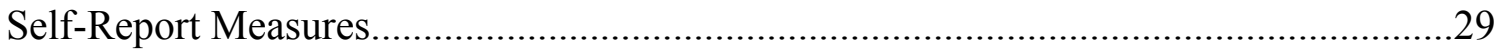




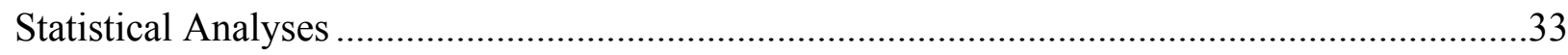

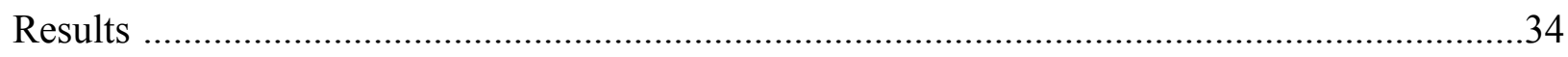

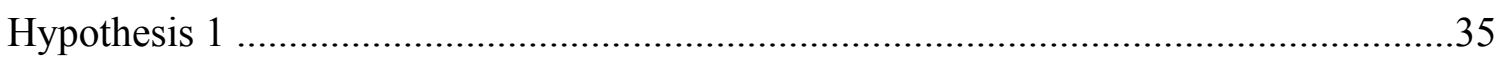

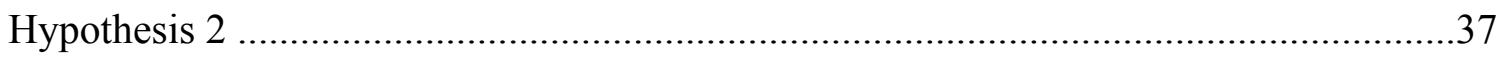

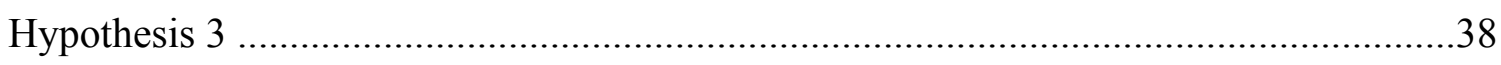

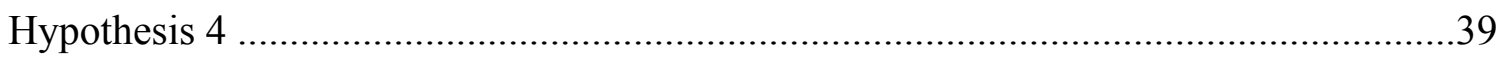

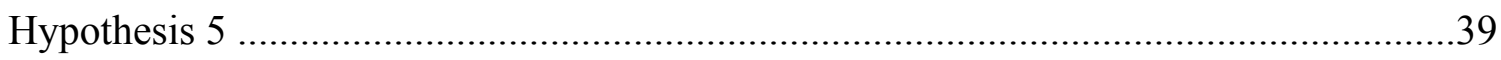

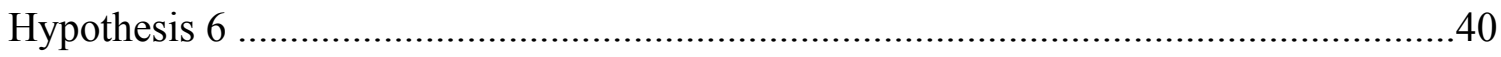

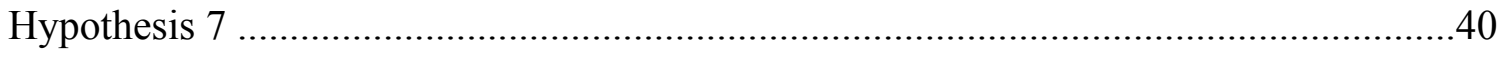

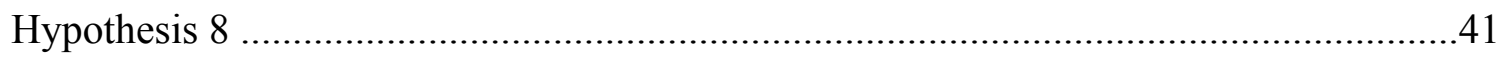

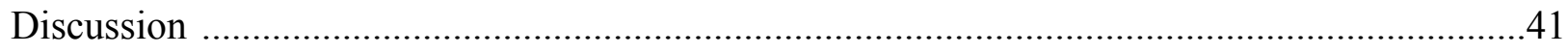

Types of ERS Associated with OCD ............................................................... 41

Cognitive Correlates of ERS ................................................................................44

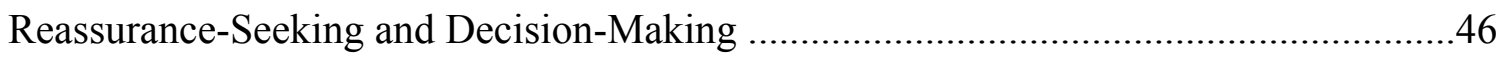

Reassurance-Seeking and Cognitive Confidence ................................................50

Intolerance of Uncertainty and Decision-Making ..................................................52

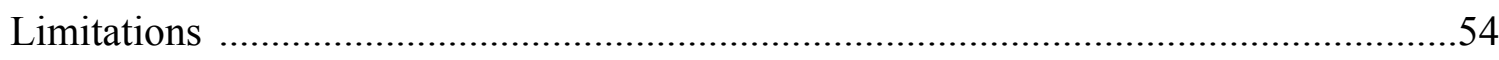

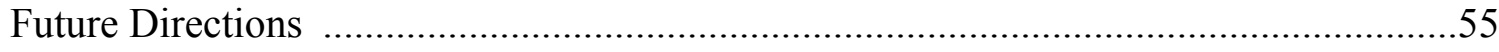

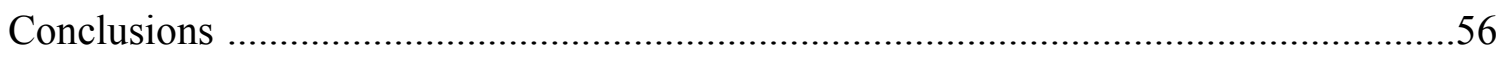

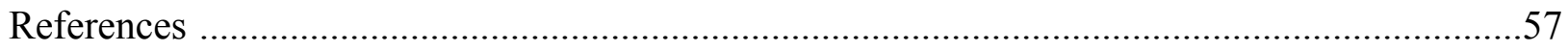




\section{LIST OF TABLES}

Table 1. Demographic characteristics for the OCD and control groups .................................19

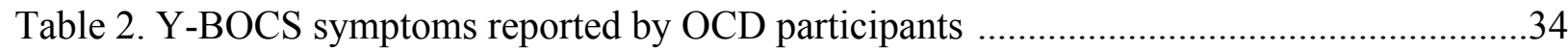

Table 3. Means and standard deviations for reported levels of ERS in response to each of the

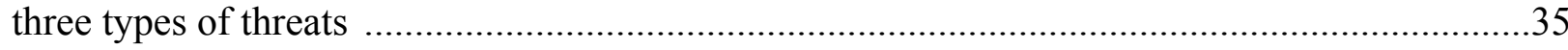

Table 4. Pearson's $r$ correlation coefficients for various domains of neurocognitive functioning

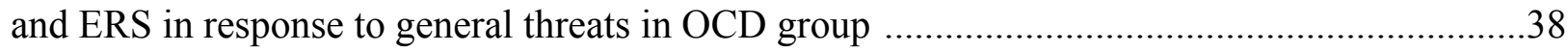




\section{REASSURANCE-SEEKING BEHAVIOUR, INTOLERANCE OF UNCERTAINTY, AND \\ NEUROCOGNITIVE FUNCTIONING IN OBSESSIVE-COMPULSIVE DISORDER \\ Introduction}

\section{Obsessive-Compulsive Disorder}

Obsessive-compulsive disorder (OCD) is a neuropsychiatric condition that affects approximately $2 \%$ to $3 \%$ of the population worldwide (Rasmussen \& Eisen, 1990), and more than one million individuals in North America (Kolada, Bland, \& Newman, 1994). OCD occurs equally in males and females (Beckstein, 2001; Lochner et al., 2005); however, males are more likely than females to have developed symptoms prior to 16 years of age (Beckstein et al., 2001). This debilitating disorder is associated with severe social and occupational impairment, and reduced quality of life (Koran, Thienemann, \& Davenport, 1996). Individuals with OCD tend to have high rates of unemployment and sick leave, and low work productivity (Honkonen et al., 2007; Kessler \& Frank, 1997; Laitinen-Krispijn \& Bijl, 2000). According to the World Health Organization, OCD is one of the top causes of disability in the world and as such, presents as a major public health concern (WHO, 1999).

OCD is defined by the presence of obsessions, compulsions, or both (Diagnostic and Statistical Manual for Mental Disorders (DSM-5); American Psychological Association (APA), 2013). Obsessions are described as recurrent intrusive thoughts that an individual experiences and attempts to suppress or neutralize by performing a compulsion. Compulsions are conceptualized as repetitive actions or thoughts that a person feels the need to perform in response to obsessions they are experiencing or rigid rules that they feel they must follow. Individuals with OCD perform these compulsions in an effort to reduce anxiety; however, the compulsions are not realistically connected to the obsessional thoughts they are experiencing 
(APA, 2013). There exist different types of obsessions and/or compulsions that individuals with OCD may experience. For example, obsessions might include content of a religious or sexual nature, or they might include thoughts about unintentionally harming others or acting on aggression, or a fear of saying something inappropriate or of making a mistake (Starcevic et al., 2012). Compulsions associated with OCD can include mental rituals such as counting or praying, or behaviours such as arranging or ordering items, hoarding belongings, washing or cleaning, or checking things such as whether the stove is turned off or whether the door is locked (Starcevic et al., 2012). This latter type of compulsion reflects compulsive checking behaviour.

Checking behaviour is the most common form of compulsion associated with OCD, and occurs in approximately $80 \%$ of individuals with the disorder (Toffolo, 2015). This type of compulsion also involves excessive feelings of responsibility for harm and beliefs about the likelihood of harm occurring (Rachman, 2002). Individuals with compulsive checking behaviour further experience persistent doubt that the perceived threat that is troubling them has been eliminated, which induces high levels of anxiety or discomfort and subsequent urges to check (Rachman, 2002). Doubt and persistent concern or worry about possible threats or harm can also lead individuals to continuously ask others for information and opinions (Parrish \& Radomsky, 2010; Radomsky, Dugas, Alcolado, \& Lavoie, 2014). This behaviour is known as excessive reassurance-seeking behaviour.

\section{Excessive Reassurance-Seeking}

Excessive reassurance-seeking (ERS) behaviour is defined as the "repeated solicitation of safety-related information from other individuals about a threatening object, situation, or interpersonal characteristic, despite having already received this information" (Parrish, 2009, p. 
3), and is conceptualized as a type of compulsive behaviour (Parrish \& Radomsky, 2010;

Rachman, 2002; Rector, Kamkar, Cassin, Ayearst, \& Laposa, 2011; Salkovskis, 1999). ERS has been associated with a particular symptom presentation of OCD.

OCD has been conceptualized as a disorder with separate symptom subtypes. The obsessions and compulsions in which an individual engages most frequently determine the symptom subtype that the individual is said to have (Williams et al., 2012). Five symptom subtypes have been identified through statistical modelling. These five subtypes include: forbidden or unacceptable thoughts, hoarding, symmetry/ordering, washing/contamination, and checking subtypes (Brakoulias, 2014; Williams et al., 2012).

ERS appears to be particularly common among individuals with the OCD checking subtype. Starcevic et al. (2012) conducted a study investigating the relationship between ERS and checking behaviour in a large sample of OCD patients. The authors used the Yale-Brown Obsessive Compulsive Scale (Y-BOCS; Goodman et al., 1989), a semi-structured interview that provides information regarding the presence and severity of different types of obsessions and compulsions. They added an item about ERS behaviour in response to obsessions to the scale to identify the presence of such behaviour among participants in the sample. The authors found that $47.9 \%$ of the OCD sample reported engaging in ERS, and that $59.7 \%$ of the participants who reported engaging in ERS also reported prominent checking compulsions on the Y-BOCS (Starcevic et al., 2012).

Rachman (2002) noted that ERS behaviour and compulsive checking behaviour are associated with similar outcomes and thus serve similar functions. It has been well established in the literature that the motivation for engaging in compulsive checking is to reduce anxiety and prevent anticipated harm (Rachman, 2002; Radomsky et al., 2014). Similarly, attempts to 
decrease anxiety and reduce the likelihood of unwanted or harmful events from occurring appear to underlie OCD patients' motivation for seeking reassurance (Cougle et al., 2012; Kobori \& Salkovskis, 2013; Parrish \& Radomsky, 2010; Rachman, 2002). However, both checking compulsions and ERS produce only temporary reductions in anxiety, following which individuals typically experience continued or worsening doubt and anxiety (Abramowitz, Franklin, \& Cahill, 2003; Rachman, 2002). In fact, ERS is associated with high levels of anxiety and sadness, particularly among individuals with OCD (Parrish \& Radomsky, 2010). Unfortunately, the temporary anxiolytic effects of these behaviours drive individuals to continue to engage in them, despite the associated negative feelings (Rachman, 2002). The negative effects associated with ERS among individuals with OCD make this behavior an important target for psychological interventions for this population.

There is an important distinction to be made between compulsive checking behaviour and ERS. In particular, whereas compulsive checking is usually performed when individuals are alone (Rachman, 2002), ERS is an interpersonal behaviour wherein the individual is seeking information and reassurance from others (Starcevic et al., 2012; Williams et al., 2012). Thus, although these behaviours serve similar functions, they are two topographically different behaviours and should be studied as such. Importantly, this means that although the research on compulsive checking behaviour is important in informing hypotheses regarding correlates of ERS behaviour, it should not be assumed that ERS shares the same correlates that have been found to be associated with compulsive checking behaviour. 


\section{Types of ERS}

Studies have shown that ERS can occur in response to a variety of different situations and thus ERS is often categorized into several different forms (Parrish \& Radomsky, 2010; Rector et al., 2011). Specifically, ERS often occurs in three different situations: (1) in response to general threats, such as the feeling that something bad is going to happen or that the stove had been left on; (2) in response to decision-related threats, or perceived risks or issues that occur at any point throughout the process of making a decision; and, (3) in response to social threats, or concerns about how others perceive you.

Parrish and Radomsky (2010) have shown that some forms of ERS may be more common in certain populations. Parrish and Radomsky (2010) compared ERS behaviour among individuals with OCD and those with depression using the Interview for Compulsive Checking and Reassurance-Seeking Behaviour Scale (ICCRS; Parrish \& Radomsky, 2010), an interview tool that these authors developed for the purpose of their study. They found that participants with OCD reported engaging in ERS most frequently in response to general threats, whereas those who were depressed were more likely to report ERS in response to perceived social threats (Parrish \& Radomsky, 2010). The authors also identified ERS in response to decision-related threats in the OCD sample (Parrish \& Radomsky, 2010).

Despite this finding demonstrating the heterogeneity of ERS behaviour, the majority of self-report measures that assess ERS were designed to address ERS in response to social threats only. These measures have been validated for use with individuals with depression (Joiner \& Metalsky, 2001; Starr \& Davila, 2008). However, the finding by Parrish and Radomsky (2010) that ERS occurs in different situations among individuals with different psychological disorders suggested that a more comprehensive measure of ERS was needed to assess for this behaviour in 
populations other than depression. In 2011, Rector and colleagues created a new ERS measure, the Reassurance Seeking Scale (RSS), that encompasses ERS behaviour in response to the three categories of threat that had been identified: general, social, and decision-making threats. Thus, in comparison to previous ERS measures that only included questions about ERS in response to social threats, this measure is more applicable to other conditions, such as OCD and anxiety disorders; the measure has been validated in these populations. Please refer to the Methods section for further information about this questionnaire.

A comprehensive examination of the relationship between OCD and these three aforementioned forms of interpersonal ERS has not yet been conducted. The study by Parrish and Radomsky (2010) described above is one of the only studies thus far to investigate types of ERS associated with OCD (Parrish \& Radomsky, 2010). Although this study contributed importantly to the literature, the authors used a semi-structured interview methodology which limits direct comparison (Parrish \& Radomsky, 2010). In contrast, research using a self-report ERS measure that has been psychometrically validated in anxiety disorder populations would allow for a direct comparison between the responses for various subscales. This type of research is needed to clarify the presence of each of the three types of ERS among individuals with OCD.

\section{Cognition}

The neurocognitive correlates of ERS among individuals with OCD have not yet been studied in the literature. However, this is an important area of research, as it could provide important information regarding the neurocognitive bases of ERS behaviour and lead to the development of targeted treatment for this behaviour. 
Cognition and OCD. OCD patients exhibit deficits in a particular set of cognitive functions, known as executive functions. Various facets of executive functioning have been shown to be impaired among these individuals, including planning, attention, and response inhibition, or the ability to suppress actions that have become habitual when they are no longer required or appropriate (Abramovitch, Abramowitz, \& Mittelman, 2013; Burdick et al., 2008; Cavedini, Gorini, \& Bellodi, 2006; Menzies et al., 2008; Olley, Mahli, \& Sachdev, 2007; Tukel et al., 2012; Verbruggen \& Logan, 2008). Deficits in working memory, or the retention and manipulation of visual and/or spatial information, another form of executive functioning, have also been identified among OCD patients (Baddeley, 2012). Other executive functioning processes have also been shown to be impaired among those with OCD including perseveration, or the unintentional repetition of an earlier response to a new situation, and set shifting, or the ability to adjust one's thinking by switching back and forth between two different concepts (Kortte, Horner, \& Windham, 2002; Sandson \& Albert, 1984). Further, OCD has been associated with impaired decision-making under ambiguous circumstances. The decision-making deficits that accompany OCD are discussed in greater detail in the Decision-Making and Indecisiveness section below.

Other cognitive deficits that are not linked to executive functioning have also been identified in OCD populations. In particular, verbal and nonverbal memory deficits and slowed processing speed have been shown to be associated with OCD (Olley et al., 2007; Shin, Lee, Kim \& Kwon, 2013; Tukel et al., 2012). Processing speed can be defined as the rate at which individuals carry out straightforward cognitive tasks (Takeuchi et al., 2011).

Cognition and ERS. Due to the lack of research on the cognitive correlates of ERS, it is difficult to formulate hypotheses regarding the areas of cognition that may be affected in OCD 
patients with ERS behaviour. However, Rachman (2002) provided compelling support for the notion that ERS and compulsive checking behaviour appear to serve similar functions among individuals with OCD. Therefore, it may be that the cognitive deficits associated with checking behaviour are also associated with ERS behaviour. Although the similarities between checking behaviour and ERS lend support to using the cognitive correlates of one behaviour to inform hypotheses regarding the cognitive correlates of the other, these two behaviours are topographically different, and the cognitive correlates of each therefore need to be examined separately. Recent research has demonstrated that each of the OCD symptom subtypes is associated with a different profile of cognitive deficits (Hashimoto et al., 2011; Lawrence et al., 2006). Individuals with the checking subtype of OCD exhibit a particular profile of cognitive impairment.

Cognition and checking behaviour. Leopold and Backenstrass (2015) performed a comprehensive meta-analysis that included all of the current literature evaluating cognitive deficits associated with the OCD checking subtype. The meta-analysis included thirteen studies from 1997 to 2015 that were conducted to investigate differences in neuropsychological performance between participants with checking and contamination/washing OCD subtypes. The results of the meta-analysis showed that checkers have significantly greater overall cognitive impairment than washers. When the specific profile of deficits for each of these two subtypes was examined, large effect sizes revealed that OCD checkers performed significantly worse than OCD washers on planning/problem-solving tasks, such as the Tower of Hanoi $(g=1.77$, $p<0.001)$, and response inhibition tasks, such the Stroop Task $(g=0.89, p<0.001)$. A medium effect size was found indicating greater impairment among OCD checkers on set shifting/cognitive flexibility tasks, such as the Trail Making Task ( $g=0.53, p<0.001)$. 
Small effect sizes were found indicating greater impairment among checkers for tests of sustained attention, such as the K-T cancellation test $(g=0.49, p=0.022)$, processing speed, such as the WAIS-R digit symbol test $(g=0.45, p=0.047)$, and encoding strategies, such as the ReyOsterrieth Complex Figure Task copy score ( $g=0.44, p=0.004$; Leopold \& Backenstrass, 2015). Additionally, small effect sizes were found for both nonverbal memory tasks ( $g=0.39, p=0.001$ ) and verbal memory tasks ( $g=0.34, p=0.027)$, indicating worse performance on these tasks among those with the checking subtype. The memory tasks included in these analyses were the California Verbal Learning Task and the Weschler Memory Scales-Revised visual reproduction.

In summary, although the sizes of the effects varied across cognitive domains, deficits in planning, response inhibition, set-shifting, encoding, processing speed, sustained attention, and non-verbal and verbal memory were all found to be significantly associated with the OCD checking subtype (Leopold \& Backenstrass, 2015). Studies that have been published since the meta-analysis by Leopold and Backenstrass (2015) have not identified any additional areas of cognitive impairment related to the OCD checking subtype.

This body of research examining the cognitive deficits associated with OCD checking symptoms provides a good indication of potential cognitive impairments related to ERS behaviour. As previously mentioned, there has been no research to date examining the relationship between cognition and ERS behaviour. Therefore, the cognitive impairments associated with the OCD checking subtype will be used to inform the neurocognitive processes that will be examined in this novel analysis. 


\section{Decision-Making and Indecisiveness}

Impaired decision-making and indecisiveness have also been linked to OCD (Kim et al., 2015; Thordarson et al., 2004). Indecisiveness can be defined as a consistent difficulty with making decisions in all areas of a person's life, whether the decisions are of little or great importance (Rassin, Muris, Franken, Smit, \& Wong, 2007). Individuals with OCD tend to report high levels of indecisiveness on self-report measures such as the Frost Indecisiveness Scale (FIS; Frost \& Shows, 1993; Thordarson et al., 2004). Although there appears to be an established link between OCD and indecisiveness, there is currently a lack of agreement in the literature about the presence of decision-making deficits in this population. Some studies have revealed impaired decision-making among individuals with OCD, whereas others have found that these individuals possess intact decision-making abilities (Cavedini, Zorzi, Piccinni, Cavallini, \& Bellodi, 2010; Chamberlain et al., 2007; Katrin Kuelz, Riemann, Zahn, \& Voderholzer, 2004; Kim et al., 2015; Nielen, Veltman, de Jong, Mulder, \& den Boer, 2002). This discrepancy may be a consequence of different decision-making constructs being tapped and different neurocognitive decisionmaking measures being used in the research.

More recent decision-making studies differentiate between two forms of decisionmaking, decision-making under risk and that under ambiguity (Brand, Labudda, \& Markowitsch, 2006; Kim et al., 2015; Pushkarskaya et al., 2015; Zhang et al., 2015). The Iowa Gambling task (IGT; Bechara, Damasio, Damasio, \& Anderson, 1994) is a measure of decision-making under ambiguity in which the outcomes of possible decisions are not defined and therefore the consequences of the decision are unknown to the participant (Brand, Recknor, Grabenhorst, \& Bechara, 2007). The Game of Dice Task (Brand et al., 2004) and the Cambridge Gambling Task (Rogers et al., 1999) are examples of measures of decision-making under risk, in which the 
participant is made aware at the beginning of the task of the possible outcomes of decisions made and their consequences (Zhang et al., 2015). Most studies show no differences in performance between OCD patients and healthy controls on tasks that measure decision-making under risk, whereas decision-making under ambiguity appears to be impaired among individuals with OCD (Zhang et al., 2015)

Decision-making under ambiguity. Numerous studies have shown that individuals with OCD make significantly more disadvantageous choices under ambiguity than do healthy controls (Kim et al., 2015; Starcke, Tuschen-Caffier, Markowitsch, \& Brand, 2009; Starcke, TuschenCaffier, Markowitsch, \& Brand, 2010; Zhang et al., 2015). Zhang et al. (2015) investigated decision-making performance on the IGT among individuals with OCD and healthy controls. Participants with OCD had difficulty avoiding disadvantageous card decks and therefore performed significantly worse than healthy controls on this task. These individuals appeared unable to take into account the information that they received from each trial to create an explicit strategy for choosing cards that would allow them to avoid losing large amounts of money under ambiguous circumstances.

ERS and decision-making. Clinically, individuals with OCD who are more indecisive or who appear to have difficulty making decisions tend to seek more reassurance from others about decisions they have to make. This may manifest as a relationship between decision-related ERS and indecisiveness, or as a relationship between decision-related ERS and impaired ambiguous decision-making. These relationships, however, have not been evaluated to date and deserve attention. 


\section{ERS and Cognitive Confidence}

Cognitive confidence is one's perception of the accuracy of his or her cognitive abilities. Thus, individuals with low cognitive confidence believe that they have poor cognitive processes such as memory, concentration and attention, and decision-making (Nedeljkovic, Moulding, Kyrios, \& Doron, 2009). Given that doubt is such a prominent feature of OCD (Rachman, 2002), researchers have investigated cognitive confidence among individuals with OCD (Nedeljkovic et al., 2009). Indeed, studies have found that individuals with OCD typically report low cognitive confidence (Hermans et al., 2008; Nedeljkovic \& Kyrios, 2007).

Cognitive confidence can also be subdivided into particular types that include confidence in one's memory, confidence in one's decision-making abilities, confidence in one's ability to focus or concentrate, and the tendency to have high standards for one's cognitive performance. The Memory and Cognitive Confidence Scale (MACCS; Nedeljkovic \& Kyrios, 2007) assesses these four domains, along with providing an overall measure of cognitive confidence.

Clinically, when individuals with OCD doubt their cognitive abilities (and thus, have low cognitive confidence), they tend to seek more reassurance from others about a variety of things, such as whether the oven was left on or whether they are making the right decision. However, the relationship between cognitive confidence and ERS has not yet been investigated in the literature.

\section{Intolerance of Uncertainty}

Individuals with OCD tend to have difficulties making decisions under ambiguous circumstances (Kim et al., 2005). A related concept is intolerance of uncertainty (IU), which can be defined as the unwillingness to accept, and difficulty handling, ambiguity. This often results 
in high levels of stress in the face of ambiguous circumstances (Jacoby, Fabricant, Leonard, Riemann, \& Abramowitz, 2013). IU is common among individuals with OCD and is often a target of psychological interventions for this population (Cougle et al., 2012; Jacoby et al., 2013).

Recent literature suggests that the best conceptual model for IU is a two-factor model that includes two different types of IU (McEvoy \& Mahoney, 2011). The first type is IU related to prospective anxiety (e.g. "one should always look ahead to avoid surprises"). This type of IU is referred to as prospective IU and involves fear of uncertainty about future events. The second form of IU is associated with inhibitory anxiety (e.g. "the smallest doubt can stop me from acting") and involves uncertainty related to one's ability to inhibit a behaviour or experience. This second type of IU is called inhibitory IU (McEvoy \& Mahoney, 2011; Meeten, Dash, Scareley, \& Davey, 2012). Discriminant validity has been demonstrated for these two subscales in past studies of individuals with anxiety and mood disorders. Specifically, the prospective IU subscale has been shown to be uniquely related to obsessive-compulsive symptoms and worry, whereas the inhibitory IU subscale has been linked to other psychological symptoms, such as social anxiety, panic, and depression (McEvoy \& Mahoney, 2011).

IU and decision-making. Pushkarskaya and colleagues (2015) found that when making decisions that required choosing between certain and uncertain monetary gains or losses, individuals with OCD tended to avoid choosing uncertain options when the probabilities of these options were ambiguous. Thus, individuals with OCD appear to have a tendency to avoid uncertainty during tasks of decision-making under ambiguity (Pushkarskaya et al., 2015). We may therefore expect individuals with OCD who exhibit high levels of IU to struggle with tasks that require ambiguous decision-making. Given the lack of past research in this area, it is unclear 
whether one or the other subscale of IU might be more closely related to decision-making impairment.

Research in healthy populations has identified a relationship between high IU and performance on ambiguous decision-making measures, such as the IGT (Bais, 2012; Luhmann, Ishida, \& Hajcaj, 2011). Specifically, participants with high IU selected cards that would provide high immediate gains, and had difficulty preventing losses on the IGT (Bais, 2012). However, no research to date has identified a clear relationship between IU and ambiguous decision-making performance in an OCD population. Investigating the relationship between these variables among individuals with OCD is very important, given that there are fundamental differences in the expression of IU among individuals with and without OCD. For example, research has shown that the strength of the relationship between IU and generalized anxiety disorder, and between IU and OCD, differ significantly (Gentes \& Ruscio, 2011). Differing expressions of IU across populations have also been demonstrated in studies that have examined the presence of the two different forms of IU among different populations. Whereas prospective IU has been shown to relate significantly to OCD and generalized anxiety disorder, inhibitory IU has been shown to be linked significantly to depression, social anxiety disorder, panic disorder, and agoraphobia (McEvoy \& Mahoney, 2011; Meeten et al., 2012). The unique cognitive features of individuals with OCD and the specific expression of IU in this population may result in differences in the relationship between decision-making and IU in this patient group as compared to healthy controls. 


\section{Research Goals}

The present study aims to identify relationships between neurocognitive functioning and several behaviours that are common among OCD patients. Specifically, this study aims to elucidate relationships between: (1) ERS in response to general threats and various areas of cognitive impairment, (2) ERS in response to decision-related threats and ambiguous decisionmaking impairment, (3) ERS and cognitive confidence, and (4) IU and ambiguous decisionmaking impairment. Further goals include clarifying the various situations in which individuals with OCD engage in ERS, and replicating the finding that OCD patients with prominent checking behaviours engage in ERS to a greater extent than those without prominent checking behaviours.

\section{Research Questions and Hypotheses}

Question 1: Do individuals with OCD seek more reassurance in response to decision-related, general, and/or social threats, as compared to healthy controls? In addition, do individuals with OCD with prominent checking behaviours engage in ERS to a greater extent than those without prominent checking behaviours?

Hypothesis 1a: It is hypothesized that individuals with OCD will report seeking greater levels of reassurance than healthy controls in response to decision-related and general threats on the Reassurance Seeking Scale (RSS). Furthermore, we hypothesize that individuals with OCD will not exhibit significantly different levels of ERS than healthy controls in response to social threats on the RSS.

Hypothesis 1b: It is hypothesized that individuals with OCD who report engaging in prominent checking behaviours on the Y-BOCS will also report engaging in ERS to a 
greater extent than individuals with OCD who do not report engaging in prominent checking behaviours on the Y-BOCS.

Question 2: Are impairments in planning, response inhibition, set-shifting, encoding, processing speed, sustained attention, and non-verbal and verbal memory significantly associated with greater ERS in response to general threats among individuals with OCD?

Hypothesis 2: It is hypothesized that deficient planning, response inhibition, setshifting, encoding, processing speed, sustained attention, and non-verbal and verbal memory will each be significantly related to greater levels of ERS in response to general threats among individuals with OCD.

Question 3: Is there a significant relationship between seeking decision-related reassurance from others and decision-making performance under ambiguity among individuals with OCD?

Hypothesis 3: It is hypothesized that there will be a significant relationship between seeking decision-related reassurance from others as reported on the RSS and objective decision-making performance on the Iowa Gambling Task among individuals with OCD.

Question 4: Is there a significant relationship between seeking decision-related reassurance from others and indecisiveness among individuals with OCD? 
Hypothesis 4: It is hypothesized that there will be a significant relationship between seeking decision-related reassurance from others on the RSS and self-reported indecisiveness on the Frost Indecisiveness Scale among individuals with OCD.

Question 5: Is there a significant relationship between overall cognitive confidence and seeking general reassurance or decision-related reassurance from others among individuals with OCD?

Hypothesis 5a: It is hypothesized that there will be a significant relationship between overall cognitive confidence on the MACCS and seeking general reassurance from others among individuals with OCD.

Hypothesis 5b: It is hypothesized that there will be a significant relationship between overall cognitive confidence on the MACCS and seeking decision-related reassurance from others on the RSS among individuals with OCD.

Question 6: Is there a significant relationship between decision-related confidence and seeking decision-related reassurance from others among individuals with OCD?

Hypothesis 6: It is hypothesized that there will be a significant relationship between reports of decision-related confidence on the MACCS and seeking decisionrelated reassurance from others on the RSS among individuals with OCD.

Question 7: Is there a significant relationship between IU and ambiguous decisionmaking performance among individuals with OCD? 
Hypothesis 7a: It is hypothesized that there will be a significant relationship between IU and ambiguous decision-making performance among individuals with OCD.

Hypothesis $7 \boldsymbol{b}$ : The relationship between ambiguous decision-making performance and both prospective and inhibitory IU will also be examined, but no specific hypotheses have been made due to the preliminary nature of these analyses.

Question 8: Is there a significant relationship between IU and indecisiveness among individuals with OCD?

Hypothesis 8a: It is hypothesized that there will be a significant relationship between IU and self-reported indecisiveness among individuals with OCD.

Hypothesis 8b: The relationship between self-reported indecisiveness on the FIS and both prospective and inhibitory IU will also be examined, but no specific hypotheses have been made due to the preliminary nature of these analyses.

\section{Method}

\section{Sample}

The sample of OCD patients in the present study consisted of 35 individuals who met DSM-5 diagnostic criteria for a primary diagnosis of OCD (APA, 2013). Forty-six percent of the OCD sample presented with one or more comorbid psychological disorders at the time of the study. The most common of these was major depressive disorder; $14 \%$ percent of OCD patients met full criteria for a Major Depressive Episode at the time of the study.

The healthy control group consisted of 37 participants who did not meet diagnostic criteria for any psychological disorder as assessed by the Mini International Neuropsychiatric 
Interview 7.0 (MINI; Sheehan \& Lecrubier, 2010). Three participants were excluded from all analyses because their self-reported scores on the Beck Depression Inventory-II (BDI-II) or Depression Anxiety and Stress Scale (DASS-21) were more than three standard deviations above the mean score for the control group, or because their scores fell within the "severe" range based on published norms for these measures. Thus, the control group employed for the present study consisted of 34 participants. The remaining control participants' scores on the BDI-II fell within the "minimal," "mild," and "moderate" categories. Further, the remaining control participants' DASS-21 scores fell within the "normal," "mild," and "moderate" ranges on the depression and anxiety subscales, and within the "normal" to "mild" ranges on the stress subscale. Age, gender, education level, and IQ for the healthy control group and for the OCD group can be found in Table 1.

Exclusion criteria for all study participants included poor English fluency and the presence of colour blindness.

Table 1. Demographic characteristics for the OCD and control groups.

\begin{tabular}{llll}
\hline & $\begin{array}{l}\text { OCD } \\
\text { Patients } \\
\mathrm{n}=35\end{array}$ & $\begin{array}{l}\text { Healthy } \\
\text { Controls } \\
\mathrm{n}=34\end{array}$ & $\begin{array}{l}\text { Difference } \\
\text { between } \\
\text { groups }\end{array}$ \\
\hline M(SD) Age in Years & $32.46(8.74)$ & $32.03(13.59)$ & $p=0.88$ \\
Female to Male Ratio ${ }^{1}$ & $6: 4$ & $5.9: 4.1$ & $p=0.92$ \\
M(SD) Education in Years & $15.43(2.12)$ & $16.38(2.47)$ & $p=0.09$ \\
M(SD) WTAR Predicted IQ & $111.94(6.95)$ & $111.88(7.11)$ & $p=0.97$
\end{tabular}

Results considered significant at $p<0.05$.

${ }^{1}$ A chi-square analysis was used to examine differences on this variable. All other analyses were done using independent samples t-tests. 


\section{Procedure}

Individuals with OCD were recruited from an ongoing collaborative study among Sunnybrook Health Sciences Centre, Hamilton Health Sciences Centre, and the Cognitive Neuroscience Lab at Ryerson University. Referrals for this study were made by mental health professionals at Sunnybrook Health Sciences Centre. Individuals were not included in the study if they met diagnostic criteria for schizophrenia, another psychotic disorder, or a substance use disorder. However, participants with comorbid depressive disorders, anxiety disorders, or OCD and related disorders, such as hoarding disorder, excoriation disorder, or trichotillomania, were not excluded due to the fact that these disorders are often comorbid. Therefore, including these participants is important for the generalizability of our results to the OCD population. Diagnoses were determined by study coordinators at Sunnybrook Health Sciences Centre or Hamilton Health Sciences Centre using the Structured Clinical Interview for DSM-5 disorders (SCID-5; APA, 2013). Prior to the use of this measure, extensive formal training on the administration and scoring of the SCID was given, which included a rigorous inter-rater reliability training program. Further, the Director of Research at the Frederick W. Thompson Anxiety Disorders Centre at Sunnybrook Health Sciences Centre, a registered clinical psychologist, oversaw all diagnoses. All clinical measures for the OCD patients were also administered at Sunnybrook Health Sciences Centre or Hamilton Health Sciences. On a separate day, participants then attended a scheduled neurocognitive assessment appointment at Ryerson University or at Hamilton Health Sciences as part of their participation in the collaborative study between these institutions.

Control participants were recruited through advertisements posted on the Ryerson University campus and in the Toronto community. Individuals who expressed interest in participating in the study as a healthy control participant were contacted by telephone to 
complete an initial screening procedure using the MINI 7.0 to rule out the presence of psychological disorders and confirm eligibility for the study. Individuals for whom inclusion criteria were met were invited to attend a testing session at Ryerson University.

All participants completed a variety of psychometrically validated neuropsychological measures and self-report questionnaires in a single session at Ryerson University or Hamilton Health Sciences Centre. All control participants were tested at Ryerson University. The assessment took, on average, three hours to complete. Measures were administered so as to reduce fatigue and to minimize interference effects. For instance, a verbally mediated measure would not have followed another verbal type task.

OCD patients received cognitive behavioural therapy through Sunnybrook Health Sciences for their participation in the study, which allowed them to participate in treatment free of charge and with a shorter waiting period than regular wait times in the Sunnybrook Anxiety Disorders Clinic. Control participants were compensated $\$ 20$ for their participation in the study. The study was approved by the Sunnybrook Health Sciences Research Ethics Board and the Ryerson University Research Ethics Board. Written informed consent was obtained from all study participants.

\section{Clinical Measures}

Several clinical measures were used with OCD patients only to assess for the presence of OCD and associated symptoms.

SCID-5. The Structured Clinical Interview for the Fifth Edition of the Diagnostic and Statistical Manual of Mental Disorders (SCID-5; First, Williams, Karg, \& Spitzer, 2014) was used to assess for the presence of OCD and comorbid psychological disorders. The SCID-5 is a 
semi-structured diagnostic clinical interview based on DSM-5 criteria. The SCID-5 has been shown to possess adequate reliability and validity (First et al., 2014).

Y-BOCS. The Yale-Brown Obsessive-Compulsive Scale (Y-BOCS) is a semi-structured interview that assesses OCD symptoms and symptom severity (Goodman et al., 1989). The YBOCS includes a checklist with 40 obsessions and 29 compulsions, and 10 items that assess symptom severity. The obsessions and compulsions that are included in the measure are organized into categories which include cleaning/washing, checking, repeating, counting, ordering/arranging, hoarding/collecting, mental compulsions, and miscellaneous. Primary obsessions and compulsions are identified using this measure. The Y-BOCS possesses good reliability and convergent validity (Woody, Steketee, \& Chambless, 1995).

\section{Screening Measures for Healthy Controls}

MINI 7.0. The Mini Neuropsychiatric Interview, Seventh Edition (MINI 7.0; Sheehan, 2014) was used to rule out DSM-5 diagnoses in the healthy control sample. The MINI 7.0 is a widely used standardized structured diagnostic interview that assesses for the presence of a variety of psychological disorders and symptoms, including persistent depressive disorder, major depressive disorder, manic and hypomanic episodes, panic disorder, agoraphobia, social anxiety disorder, obsessive-compulsive disorder, posttraumatic stress disorder, alcohol use disorder, substance use disorders, anorexia nervosa, bulimia nervosa, binge-eating disorder, generalized anxiety disorder, specific phobia, body dysmorphic disorder, illness anxiety disorder, and psychotic symptoms. Participants are asked a variety of questions about psychological symptoms and are instructed to provide "yes" or "no" answers. Previous versions of the MINI have been shown to have excellent inter-rater and test-retest reliabilities and adequate convergent validity 
with the SCID, a well-validated, comprehensive clinical measure (Sheehan et al., 1997; Sheehan et al., 1998). Although comparable psychometric data is not yet available for the newer MINI 7.0, the changes that were made for the seventh edition of the MINI were based directly on the diagnostic criteria for the DSM-5 (Sheehan \& Lecrubier, 2010; Sheehan, 2014).

\section{Neurocognitive Measures}

\section{Premorbid Intelligence}

WTAR. The Wechsler Test of Adult Reading (WTAR) provides an estimate of premorbid intelligence level (Holdnack, 2001). For this task, the participant is instructed to read out loud a printed list of 50 words. The number of words that an individual pronounces correctly are added up and compared against normative data to predict the individual's full scale intelligence quotient (IQ). The WTAR possesses good reliability and predictive validity (Holdnack, 2001).

\section{Memory}

California Verbal Learning Test. The California Verbal Learning Test (CVLT) is a neurocognitive measure of verbal memory, and includes free recall, cued recall, and recognition trials (Delis, Kramer, Kaplan, \& Thompkins, 1987). First, the participant is read a list of 16 words that fall into four different categories and asked to recall as many items as he or she can from the list. This is repeated four additional times, with participants recalling as many items as possible on each trial. After the fifth trial, the participant is read a second list of 16 interference words and asked to recall items from this second list only. Next, the participant is instructed to recall as many items as possible from the initial list for the short delay free recall trial. The participant is then asked again to recall words from this same list, but this time the assessor cues 
the participant by naming each of the four categories of words that were included on the list. A twenty-minute delay is then initiated.

Following the delay, the second free recall and cued recall trials are presented. A recognition trial is then presented wherein the participant is read a list of 40 words and asked to state whether or not each item was included on the initial list. Some of the words in this 40 -word list are from the first list, some are from the interference list, and some are words that have not yet been presented on this test. Finally, there is a 10-minute delay, following which the participant is read two words at a time and asked to state which of these two words was on the first list. This last component of the CVLT is a validity measure. For the present study, all participants' scores fell below the cut-off for invalid responses, and therefore all data from the CVLT was considered valid.

The verbal memory outcome measure for the present study is the sum of the items correctly recalled on the delayed recall trial. The CVLT demonstrates good construct validity and test-retest reliability (Delis, Kramer, \& Kaplan, 1988; Woods, Delis, Scott, Kramer, \& Holdnack, 2006).

\section{Rey-Osterrieth Complex Figure Test. The Rey-Osterrieth Complex Figure Test} (RCFT) is a paper and pencil neurocognitive measure of visuospatial encoding and memory (Osterrieth, 1944). The participant is asked to copy a complex figure onto a sheet of paper as accurately as possible. For the initial copy trial, the length of time it takes the participant to draw the figure is recorded. Immediate and delayed recall trials are presented three and 20 minutes, respectively, after the initial copying task. On each of these trials, the participant is asked to draw the initial figure from memory. Following the recall trials, there is a recognition trial in which the participant is presented with a variety of figures printed on four pages of paper. The participant is 
asked to circle the figures that were part of the larger initial figure (i.e., presented in the same orientation as they were on that larger figure). Accuracy of each drawing is scored using the 36point system developed by Lezak (1995). Scores on the copy trial of the RCFT represent the outcome variable for encoding. The non-verbal memory outcome variable is the score of the drawing produced on the delayed recall trial. The RCFT possesses adequate construct validity, test-retest reliability, and inter-rater reliability (Meyers \& Meyers, 1995).

\section{Decision-Making}

Iowa Gambling Task. The Iowa Gambling Task (IGT) is a neurocognitive measure of decision-making under ambiguity (Bechara et al., 1994). This computerized task includes four decks of cards from which the participant must choose one card at a time by clicking on the top of the deck. The participant is given a credit of $\$ 2000$ of pretend money, represented by a green bar on the computer screen, prior to beginning the task. A red bar of the same size is also presented on the screen, and this represents a reminder of how much money the participant borrowed to begin the task. The participant is read a list of instructions regarding the task, which includes the fact that he or she is to treat the money in the game as if it were his or her real money. The examiner explains that the decks differ in terms of how advantageous they are, and that it is therefore possible to succeed by avoiding the decks that are worse. Each time the individual picks a card, the computer informs him or her that he or she has won a sum of money.

On some trials, the participant will also be informed that he or she has lost a sum of money. Each monetary win and loss is associated with a subsequent increase or decrease, respectively, in the length of the green bar on the computer screen. The participant must complete a total of 100 trials. 
The participant is not informed that two of the decks involve moderate gains and moderate losses, whereas the other two decks involve high gains and high losses. Therefore, there is ambiguity associated with the outcome of each trial. Participants must learn which decks are associated with greater risk if they are to do well on this task (Brand et al., 2006). Participants' responses are analyzed by comparing the total number of cards they picked from the advantageous versus disadvantageous decks. The outcome variable for this task is the amount of money the participant has remaining at the end of the game; a larger amount of money indicates that a greater number of cards were chosen from the advantageous decks and is thus indicative of better decision-making performance. The 100 trials administered are typically separated into five sets of trials, and the net total sum of money the participant receives or loses during each of these five sets of trials are also outcome variables for this task. The IGT possesses adequate construct validity (Buelow \& Suhr, 2009).

\section{Sustained Attention}

Ruff 2\&7 Selective Attention Test. The Ruff 2\&7 Selective Attention Test is a paper and pencil task that measures sustained attention (Ruff, Niemann, Allen, Farrow, \& Wylie, 1992). Sustained attention is the ability to maintain consistent performance. Participants are given the Ruff $2 \& 7$ booklet which includes blocks with either number or letter distractor stimuli along with $2 \mathrm{~s}$ and $7 \mathrm{~s}$, and are instructed to cross out all the $2 \mathrm{~s}$ and $7 \mathrm{~s}$ in order, as quickly as they can, in each block. After 15 seconds, the assessor instructs the participant to move on to the next block. There are a total of 20 blocks to complete. The primary outcome variable for this measure is the difference between the total speed score and the total accuracy score. The Ruff $2 \& 7$ task has high internal consistency, test-retest reliability, and construct validity (Ruff et al., 1992). 


\section{Planning}

Tower of London. The Tower of London (TOL) planning task is a neurocognitive measure of spatial planning (Shallice, 1982). The TOL involves two peg boards, one for the assessor and one for the participant. Each board has three pegs of varying lengths with coloured beads on it. The participant is instructed that he or she must move the beads on his or her board to make each of the patterns that the assessor makes. The participant is also told the maximum number of beads that can be placed on each peg, and that he or she is only allowed to move one bead at a time. The assessor then makes a series of patterns on his or her board and instructs the participant to copy them, keeping track of the number of moves the participant makes to complete the pattern. The assessor also notes the length of time it takes the participant to initiate problem-solving, as well as the total problem-solving time for each pattern. Planning accuracy, the outcome variable for this measure, is determined by evaluating the number of trials that a participant completes using the minimum number of moves (Shallice, 1982). The TOL possesses adequate reliability and construct validity (Culbertson \& Zillmer, 1998; Shallice, 1982).

\section{Response Inhibition}

Stop Signal Task. The Stop Signal Task (SST) is a computerized neurocognitive measure of motor response inhibition (Logan, Cowan, \& Davis, 1984). The task involves two types of trials, go trials and stop trials. The go trials involve a simple choice reaction time task. Participants are initially presented with a fixation point that appears on a computer screen for 500ms. Upper case " $\mathrm{X}$ " and "O" letters are then presented in the centre of the screen for 1000 ms, followed by a 2000 ms blank screen. Participants are instructed to respond as quickly and accurately as possible by pressing the " $\mathrm{X}$ " key on the computer keyboard when an " $\mathrm{X}$ " appears, or the period key on the keyboard when an "O" appears. 
On $25 \%$ of the trials, a $1000 \mathrm{~Hz}$ stop signal is randomly presented following the letter. Participants are instructed not to press the letter's corresponding key when this signal is presented. These trials make up the stop trials. The stop signal delay is initially set at $250 \mathrm{~ms}$, and a tracking algorithm converges on the stop signal delay at which individuals are able to inhibit $50 \%$ of responses. The later the tone is presented, the more difficult it is to inhibit the response.

The SST consists of eight blocks of 32 trials. Twenty-four of these trials are go trials without a stop signal, while eight of the trials are stop trials. The outcome variable for the SST is stop signal reaction time, which is the latency between the appearance of the stop signal and the initiation of the stop process. This is calculated by subtracting the mean delay from the mean reaction time. The SST has been validated using various responses, including button presses, typing, and eye movements, and in a variety of populations. Test-retest reliability values indicate that performance on the SST is stable over time (Schachar et al., 2004).

\section{Set shifting}

Trail Making Test. The Trail Making Test (TMT) is a paper and pencil neurocognitive measure of information processing speed, working memory, and task switching (Kortte, Horner, \& Windham, 2002). The task includes two parts. The materials for part A consist of a sheet of paper with the numbers 1 through 25 printed on it, and the materials for part B include a sheet of paper with the numbers 1 through 13 and the letters A through L printed on it. In part A, the participant must connect the numbers in order as quickly as possible, without lifting his or her pencil from the page. In part B, the participant must connect the numbers and letters in order as

quickly as possible, alternating between number and letter. The outcome variables for part A and part B are the length of time it takes the participant to complete each part of the task. The TMT 
demonstrates adequate construct validity and high inter-rater reliability (Fals-Stewart, 1992; Sanchez-Cubillo et al., 2009).

\section{Processing Speed}

Symbol Digit Modalities Test. The Symbol Digit Modalities Test (SDMT) is a brief paper and pencil task that measures information processing speed (Smith, 1982). At the top of the test form, there is a key where digits one through nine are each matched with a corresponding symbol. Beneath the key, the page is filled with rows of symbols. Participants are instructed to write the corresponding number from the key below each of these symbols. They are told to do so by matching the symbols in each row in order, from left to right, without skipping any symbols, and to go as quickly as they can. The number of symbols matched correctly in 90 seconds is the outcome variable for the SDMT. The SDMT demonstrates adequate test-retest reliability and correlates highly with the WAIS Digit Symbol test, another widely used measure of information processing speed (Sheridan et al., 2006).

\section{Self-Report Measures}

Reassurance Seeking Scale. The Reassurance Seeking Scale (RSS) is a self-report measure that assesses the degree to which participants seek reassurance in response to a variety of situations (Rector et al., 2011). Participants indicate on a 5-point Likert scale the frequency with which they seek reassurance in each situation. The scale ranges from 1 or "not at all" to 5 or "extremely." The situations that are presented on the RSS are divided into three categories: decision-making threats, general threats, and social threats. The outcome measures for this scale include the total scores for ERS in response to decision-making threats, general threats, and social threats. The RSS possesses good internal consistency and convergent validity with anxiety 
symptoms on the Beck Anxiety Scale (BAI; Beck \& Steer, 1990) and the Depression Anxiety and Stress Scale (DASS; Lovibond \& Lovibond, 1995) among anxiety disorder populations (Rector et al., 2011). Internal consistency for the RSS ranged from $\alpha=0.88$ to $\alpha=0.93$ in a sample of individuals with OCD and other anxiety disorders (Rector et al., 2011).

Memory and Cognitive Confidence Scale. The Memory and Cognitive Confidence Scale (MACCS) is a self-report scale that measures four areas of cognitive confidence: confidence in one's general memory abilities, confidence in one's decision-making abilities, confidence in one's ability to focus or concentrate, and one's standards for cognitive performance (Nedeljkovic \& Kyrios, 2007). The scale includes a total of 28 items and participants indicate their level of agreement with each on a 5-point scale that ranges from "strongly disagree" to "strongly agree." Outcome measures for this scale are the total scores on each of the four areas of cognitive confidence, along with an overall cognitive confidence score made up of the sum of each of these subscales. The MACCS possesses good test-retest reliability in both clinical and community samples (Nedeljkovic \& Kyrios, 2007). The internal consistency for this measure is excellent $(\alpha=0.92$; Nedeljkovic $\&$ Kyrios, 2007).

Frost Indecisiveness Scale. The Frost Indecisiveness Scale (FIS) is a 15-item self-report scale that measures indecisiveness by assessing one's tendency to postpone or struggle with decision-making (Frost \& Shows, 1993). Participants indicate the degree to which they endorse a variety of scenarios regarding making decisions using a 5-point Likert scale that ranges from "strongly disagree" to "agree." The outcome measure for this scale is the sum of all responses after reverse-coding items that are indicative of no difficulties with making decisions. Research supports the FIS as a valid and reliable measure of self-reported decision-making difficulty (Frost \& Shows, 1993). The measure has also been shown to have good internal consistency 
$(\alpha=0.90 ;$ Frost $\&$ Shows, 1993). Good test-retest reliability and predictive validity have also been demonstrated for this measure (Rassin, Muris, Franken, Smit, \& Wong, 2007).

Intolerance of Uncertainty Scale-12. The Intolerance of Uncertainty Scale-12 (IUS-12) is a self-report questionnaire that measures the extent to which participants are able to tolerate uncertainty and ambiguity. Participants indicate their level of agreement with each of the 12 items on a 5-point Likert scale that ranges from "not at all characteristic of me" to "entirely characteristic of me" (Carleton, Norton, \& Asmundson, 2007). This measure is an abbreviated version of the original Intolerance of Uncertainty Scale (IUS), a 27-item self-report measure that possesses high internal consistency and test-retest reliability (Buhr \& Dugas, 2002; Freeston, Rheaume, Letarte, Dugas, \& Ladouceur, 1994). The IUS-12 is highly correlated with the original IUS and also demonstrates high internal consistency, and good test-reliability, discriminant validity, and predictive validity (Boelen \& Carleton, 2012; Helsen, Van den Dussche, Vlaeyen, \& Goubert, 2013; Khawaja \& Yu, 2010). The IUS-12 has also been shown to have excellent internal consistency, $\alpha=0.93$ in a sample of OCD patients (Jacoby et al., 2013). The measure includes two subscales: one that includes the sum of all items related to prospective IU, and the other which consists of the sum of all items related to inhibitory IU. An overall score can be obtained by summing the scores from these two subscales (Carleton et al., 2007).

Beck Depression Inventory-II. The Beck Depression Inventory-II (BDI-II) is a selfreport measure that assesses depressive symptoms (Beck, Steer, \& Brown, 1996). The measure is derived from DSM-IV major depressive disorder criteria. The measure includes 21 items about various symptoms of depression. Each of these 21 items have four to seven statements that represent varying degrees to which individuals experience the symptom, from a lack of these symptoms (rated as 0 ) to the persistence of severe symptoms (rated as 3 ). The participant is 
instructed to circle the item that best fits their experience over the past two weeks. The BDI-II has been shown to be sensitive to picking up on depressive symptoms (Subica et al., 2014). In addition, the measure is valid and reliable for use as a screening measure (Wang \& Gorenstein, 2013). The measure also demonstrates good internal consistency in non-clinical samples ( $\alpha=0.93$; Beck, Steer, \& Brown, 1996).

This questionnaire was included to provide a quantitative supplement to the MINI 7.0 to ensure eligibility criteria among healthy controls. Individuals with scores greater than three standard deviations from the mean for the control group or whose scores fell within the "severe" range of symptoms based on published norms for this measure (Beck, Steer, \& Brown, 1996) were excluded from all analyses.

Depression Anxiety and Stress Scale-21. The Depression, Anxiety, and Stress Scale-21 (DASS-21) measure is a questionnaire that assesses symptoms of depression, anxiety, and stress (Lovibond \& Lovibond, 1995). Participants are instructed to rate 21 statements about various symptoms on a 4-point scale. A score of 0 indicates that the item never applies, and a score of 3 indicates that the item applies very much or most of the time. Three scores are derived from participants' reports on this measure: a depressive symptoms score, an anxiety symptoms score, and a stress symptoms score (Henry \& Crawford, 2005). This measure has been shown to have good reliability and internal consistency in a non-clinical sample, $\alpha=0.88$ (Antony, Bieling, Cox, Enns, \& Swinson, 1998; Henry \& Crawford, 2005; Samani \& Joukar, 2007). The measure has also been shown to have adequate construct validity in assessing measures of depression, anxiety, and stress (Henry \& Crawford, 2005). The DASS-21 was included to further rule out control participants with high levels of depression, anxiety, and stress. 
The measure was included to provide a quantitative supplement to the MINI 7.0 to ensure eligibility criteria among healthy controls. Individuals with scores greater than three standard deviations from the mean for the control group or whose scores fell within the "severe" range of symptoms based on published norms for this measure (Lovibond \& Lovibond, 1995) were excluded from all analyses.

\section{Statistical Analyses}

The data were analyzed using the Statistical Package for Social Sciences, Version 20 (SPSS; Nie, Bent, \& Hull, 1970). Two-tailed independent samples t-tests were conducted to investigate Hypotheses 1a and 1b. Correlational analyses were used to examine Hypothesis 2. Hypotheses 3, 4, 5a, 5b, 6, 7a, 7b, 8a, and 8b were examined using linear regression analyses. Results were considered significant at a level of $p<0.05$.

There were some missing data due to questionnaires not being filled out correctly and to issues with neurocognitive task administration. However, overall, the amount of missing data was minimal (i.e., no variable had greater than $2.8 \%$ missing data) and therefore missing values were not replaced for the purposes of this study.

For all participants, outlier scores on neurocognitive variables were removed from analyses. Outliers were defined as any score that fell three or more standard deviations away from the mean for that group (Osborne \& Overbay, 2004).

As previously noted in the Methods section, all data from healthy control participants with BDI-II or DASS-21 scores three or more standard deviations from the control group means for these measures, or with scores in the "severe" range, were removed from all analyses. 


\section{Results}

The OCD sample presented with various obsessions and compulsions. Reported Y-BOCS symptoms for each symptom dimension are presented in Table 2. The symptom presentations in the present study demonstrate adequate consistency with those identified in past studies of OCD (Lawrence et al., 2006; Rosario-Campos et al., 2006; Toffolo, 2015). Symptom severity scores on the Y-BOCS were also in line with the typical severity levels among OCD patients, based on past studies $(M=23.6, S D=5.19$; Goodman et al., 1989; Shin et al., 2013). Further, $65.72 \%$ of individuals in the OCD group reported that they were currently using prescribed psychotropic medication for OCD, anxiety, or mood at the time of the study, whereas $34.28 \%$ of participants reported that they were not taking any of these medications. This rate of psychotropic medication use in OCD participants is similar to rates of use in past studies (Abramovitch et al., 2013).

Table 2. Y-BOCS symptoms reported by OCD participants.

\begin{tabular}{ll}
\hline Obsessive/compulsive symptom domain & $\begin{array}{l}\text { Percent of OCD participants in the sample } \\
\text { who reported symptoms within this } \\
\text { domain (current or past) }\end{array}$ \\
\hline Aggressive & $60 \%$ \\
Sexual/Religious/Forbidden Thoughts & $68.57 \%$ \\
Checking & $82.86 \%$ \\
Ordering/Symmetry & $65.71 \%$ \\
Contamination/Cleaning & $85.71 \%$ \\
Hoarding & $48.57 \%$ \\
\hline
\end{tabular}




\section{Hypothesis 1}

To investigate Hypothesis 1a, independent samples t-tests were conducted to determine whether there is a significant difference between OCD patients and healthy controls in the extent to which they report engaging in each of the three forms of ERS. Analyses demonstrated a significant difference between OCD patients and healthy controls in the degree to which they reported seeking reassurance in response to general threats $(t(66)=-5.62, p<0.001)$, decisionrelated threats $(t(67)=-3.28, p=0.002)$, and social threats $(t(67)=-2.81, p=0.006)$. Means and standard deviations for reported levels of ERS in response to each of the three types of threats can be found in Table 3 .

Table 3. Means and standard deviations for reported levels of ERS in response to each of the three types of threats.

\begin{tabular}{lll}
\hline Type of ERS & $\begin{array}{l}\text { OCD M(SD) } \\
\mathrm{n}=35\end{array}$ & $\begin{array}{l}\text { Healthy Controls M(SD) } \\
\mathrm{n}=34\end{array}$ \\
\hline General Threats* & $28.32(9.23)$ & $16.88(7.48)$ \\
Decision-Related Threats* & $40.80(14.71)$ & $30.35(11.54)$ \\
Social Threats* & $23.09(8.19)$ & $17.73(7.57)$ \\
\hline$*=p<0.05$ & &
\end{tabular}

Supplementary analyses were conducted to investigate Hypothesis 1a while excluding individuals from the OCD group who also met diagnostic criteria for a major depressive episode $(n=5)$. These supplementary analyses were done to examine the role that comorbid depression has in the type of ERS in which individuals with OCD engage, given that previous research has demonstrated that ERS in response to social threats is commonly exhibited by individuals with depressive disorders (Parrish \& Radomsky, 2010). Significant differences remained in the degree 
to which individuals with OCD and healthy controls reported engaging in ERS in response to general threats $(t(61)=-5.05, p<0.001)$, decision-related threats $(t(54.05)=-2.68, p=0.01)$, and social threats $(t(62)=-2.45, p=0.017)$. Unequal variances were assumed for the analysis between decision-related threats among OCD participants and healthy controls. Homogeneity of variance was assumed for all other analyses.

Supplementary analyses were also done to investigate hypothesis 1a while excluding individuals from the healthy control group who reported moderate levels of anxiety on the DASS-21 $(n=6)$. These analyses were done to determine whether the presence of anxiety symptoms among healthy controls may have played a role in the observed differences between the OCD and control groups with respect to ERS behaviour. Significant differences were found in the degree to which individuals with OCD and healthy controls with low levels of anxiety reported engaging in ERS in response to general threats $(t(60)=-6.40, p<0.001)$, decisionrelated threats $(t(59.90)=-3.98, p<0.001)$, and social threats $(t(61)=-3.96, p<0.001)$.

To investigate Hypothesis $1 \mathrm{~b}$, independent samples t-tests were done to examine differences in ERS behaviour among individuals with OCD who reported checking behaviour as a primary compulsion on the Y-BOCS and those who did not report checking as a primary symptom. Fourteen out of 35 participants reported engaging primarily in checking compulsions on the Y-BOCS. No significant differences were found between individuals with and without primary checking behaviour with respect to the degree to which they reported engaging in ERS in response to general, $(t(32)=0.768, p=0.448)$, decision-related, $(t(33)=1.759, p=0.088)$, and social threats, $(t(33)=0.033, p=0.974)$. Supplementary analyses revealed that checking symptoms on the Y-BOCS co-occurred with ERS in response to general threats at a rate of $95.65 \%$. 


\section{Hypothesis 2}

For Hypothesis 2, relationships between ERS in response to general threats and various domains of neurocognitive functioning were examined using Pearson's correlations. Specifically, the relationship between ERS in response to general threats, and (1) the number of problems that individuals were able to solve correctly in the minimum number of moves on the Tower of London Task (a measure of planning ability), (2) stop signal reaction time on the Stop Signal Task (a measure of response inhibition), (3) performance on the Trail Making B Task (a measure of set shifting), (4) total difference between participants' speed and accuracy on the Ruff $2 \& 7$ (a measure of sustained attention), (5) performance on the Symbol Digit Modalities Task (a measure of processing speed), (6) performance on the copy trial of the Rey Complex Figure Task (RCFT; a measure of encoding), (7) performance on the delayed recall trial of the RCFT (a measure of non-verbal memory), and (8) performance on the long-delay recall trial of the of the California Verbal Learning Task (a measure of verbal memory). None of the eight correlations were found to be significant. The correlation coefficients from the analyses are presented in Table 4. 
Table 4. Pearson's $r$ correlation coefficients for various domains of neurocognitive functioning and ERS in response to general threats in OCD group.

\begin{tabular}{|c|c|c|c|c|c|c|c|c|}
\hline & $\begin{array}{l}\text { Planning: } \\
\text { Tower of } \\
\text { London } \\
\text { Task }\end{array}$ & $\begin{array}{l}\text { Response } \\
\text { Inhibition: } \\
\text { Stop Signal } \\
\text { Task }\end{array}$ & $\begin{array}{l}\text { Set } \\
\text { Shifting: } \\
\text { Trail } \\
\text { Making } \\
\text { Task B }\end{array}$ & $\begin{array}{l}\text { Sustained } \\
\text { Attention: } \\
\text { Ruff } 2 \& 7\end{array}$ & $\begin{array}{l}\text { Processing } \\
\text { Speed: } \\
\text { Symbol } \\
\text { Digit } \\
\text { Modalities } \\
\text { Task }\end{array}$ & $\begin{array}{l}\text { Encoding: } \\
\text { RCFT } \\
\text { Copy Trial }\end{array}$ & $\begin{array}{l}\text { Non- } \\
\text { Verbal } \\
\text { Memory: } \\
\text { RCFT } \\
\text { Delayed } \\
\text { Recall }\end{array}$ & $\begin{array}{l}\text { Verbal } \\
\text { Memory: } \\
\text { CVLT } \\
\text { Delayed } \\
\text { Recall }\end{array}$ \\
\hline $\begin{array}{l}\text { ERS in } \\
\text { Response } \\
\text { to } \\
\text { General } \\
\text { Threats }\end{array}$ & -0.181 & 0.260 & 0.052 & 0.032 & -0.059 & -0.147 & 0.080 & 0.070 \\
\hline
\end{tabular}

\section{Hypothesis 3}

Linear regression analyses were performed to investigate Hypothesis 3. A significant negative relationship was found between ERS in response to decision-related threats and participants' final sum of money on the IGT $(b=-49.97, S E=18.41, t=-2.72, p=0.010)$ among OCD patients. This indicates that as ERS in response to decision-related threats increases, performance on the IGT decreases, indicating impairment. The effect size for this relationship was $\beta=-0.427$. Supplementary linear regression analyses were done to examine the relationship between these two variables at various points throughout the IGT. On the first set of trials, no significant relationship was found between ERS in response to decision-related threats and participants' net performance on these trials as measured by the net total money $(p>0.05)$. On the second $(b=-0.55, S E=0.17, t=-3.33, p=0.002)$, fourth $(b=-0.46, S E=0.16, t=-2.84, p=0.008)$, and fifth $(b=-0.43, S E=0.15, t=-2.88, p=0.007$ ) sets of trials, significant negative relationships were observed between ERS in response to decision-related threats and participants' net total. 
The relationship between ERS in response to decision-related threats and participants' net total was approaching significance on the third set of trials $(b=-0.36, S E=0.20, t=-1.87, p=0.071)$.

A supplementary independent samples t-test was performed to investigate the difference between OCD patients' $(M=-590.29, S D=1720.62)$ and healthy controls' $(M=175.74$, $S D=1664.96$ ) overall performance on the IGT ambiguous decision-making task. The difference between the total amount of money each group had remaining at the end of the IGT was approaching significance, $t(67)=1.88, p=0.065$. The difference between OCD patients' and

healthy controls' performance was also examined at various levels of the IGT, and a significant difference was found between the two groups' performance on the fifth set of trials $(t(67)=$ $2.06, p=0.044)$, but not on the first four sets of trials $(p>0.05)$. OCD patients' scores were significantly lower than those of the healthy controls on the fifth set of trials.

\section{Hypothesis 4}

Linear regression analyses were performed to investigate Hypothesis 4. A significant positive relationship was identified between ERS in response to decision-related threats and indecisiveness on the FIS among OCD patients $(b=0.69, S E=0.09, t=7.33, p<0.001)$. This indicates that as reported levels of decision-related ERS increase, reported levels of indecisiveness increase as well. The effect size for this relationship was $\beta=0.792$.

\section{Hypothesis 5}

Hypothesis 5a was tested using a linear regression analysis. The relationship between overall cognitive confidence on the MACCS and ERS in response to general threats among OCD patients was not significant $(p>0.05)$. Hypothesis $5 \mathrm{~b}$ was also tested using a linear regression 
analysis. There was a significant positive relationship between overall cognitive confidence on the MACCS and ERS in response to decision-related threats among OCD patients $(b=6.19$, $S E=1.70, t=3.65, p=0.001)$. Thus, as reported levels of cognitive confidence decrease (as measured by an increase in scores on the MACCS), reported levels of ERS in response to decision-related threats increase.

\section{Hypothesis 6}

Linear regression analyses were used to investigate Hypothesis 6. A significant positive relationship was found between decision-related cognitive confidence on the MACCS and ERS in response to decision-related threats among OCD patients $(b=9.33, S E=1.60, t=5.85$, $p<0.001$ ). This reveals that as reported levels of decision-related confidence decrease (as measured by an increase in scores on the MACCS), reported levels of ERS in response to decisions increase.

\section{Hypothesis 7}

To investigate Hypotheses $7 \mathrm{a}$ and $7 \mathrm{~b}$, linear regression analyses were performed. Results did not reveal a significant relationship between overall level of IU and ambiguous decisionmaking as measured by participants' total money at the end of the IGT $(p>0.05)$. Furthermore, no significant relationship emerged between prospective IU, as measured by the prospective subscale of the IUS-12, and ambiguous decision-making performance among OCD patients $(\mathrm{p}>0.05)$. However, a significant negative relationship was observed between inhibitory IU, as measured by the inhibitory subscale of the IUS-12, and ambiguous decision-making performance $(b=-151.05, S E=55.62, t=-2.72, p=0.01)$ among OCD patients. Thus, as inhibitory IU scores 
increase, ambiguous decision-making performance decreases, indicating greater decision-making impairment. The effect size for this relationship was $\beta=-0.427$.

\section{Hypothesis 8}

Linear regression analyses were performed to investigate Hypotheses $8 \mathrm{a}$ and $8 \mathrm{~b}$. A significant positive relationship was identified between overall level of IU, as measured by the total IUS-12 score, and self-reported indecisiveness as measured by the FIS $(b=0.80, S E=0.22, t=$ 3.70, $p=0.001$ ) among OCD patients. This indicates that as reported levels of overall IU increase, reported levels of indecisiveness increase as well. The relationship between prospective IU, as measured by the prospective subscale of the IUS-12, and indecisiveness as measured by the FIS among OCD patients was not found to be significant $(p>0.05)$. A significant positive relationship was found between inhibitory IU as measured by the inhibitory subscale of the IUS-12, and indecisiveness as measured by the FIS among OCD patients $(b=1.90, S E=0.32, t=5.87$, $p<0.001$ ). The effect size for this relationship was $\beta=0.720$. Thus, as reported levels of inhibitory IU increase, reported levels of indecisiveness also increase.

\section{Discussion}

\section{Types of ERS Associated with OCD}

Consistent with Hypothesis 1a, significant differences were identified in the degree to which individuals with $\mathrm{OCD}$ and healthy controls reported seeking reassurance in response to all three types of threats (i.e., general, decision-related, and social). ERS in response to general threats such as a concern that the stove has been left on has previously been shown to be the most common type of ERS exhibited by individuals with OCD (Parrish \& Radomsky, 2010). 
Past research has demonstrated that individuals with OCD tend to accumulate higher levels of information than healthy controls prior to making decisions (Banca et al., 2015). Results from the present study indicate that individuals with OCD also appear to seek higher levels of verbal information or opinions about decisions they are making than do healthy controls.

The OCD group in the present study reported also greater levels of ERS in response to social threats on the Reassurance-Seeking Scale. It is possible that this finding is related to the presence of comorbid psychological disorders among OCD patients in the present study. Past research has shown that ERS in response to social threats is most frequently reported in depression samples (Parrish \& Radomsky, 2010). The OCD group for the present study included five individuals who met criteria for a major depressive episode at the time of the study (i.e., $14 \%$ of the OCD group). To investigate the role that the presence of a major depressive disorder played in the results, supplementary analyses were conducted to examine whether individuals with OCD who did not have major depressive disorder also reported engaging in significantly greater levels of ERS than healthy controls. Interestingly, individuals with OCD and without depression reported engaging in ERS in response to general, decision-related, and social threats to a significantly greater degree than healthy controls as well. Individuals with OCD therefore appear to seek greater levels of reassurance than healthy controls in all of these circumstances, regardless of the presence of depression.

It is possible that other factors are playing a role in this observed result. For example, it could be that medication use by individuals with OCD is linked to ERS behaviour. A complete analysis of the medication use by OCD participants was not done for the present study. However, $34.28 \%$ of these participants reported that they were not taking any medication for OCD, anxiety, or mood at the time of the study. Past research has shown that anti-anxiety medication 
does not appear to impact neurocognitive functioning among individuals with OCD (Abramovitch et al., 2013; Wingen, Bothmer, Langer, \& Ramaekers, 2005). However, no research to date has examined the impact of these medications on ERS behaviour, and this should therefore be a focus of future research studies. Despite the lack of research on this topic, one might expect that ERS behaviour would be reduced among those with OCD who are using these medications, given that anxiety medications target symptoms of OCD and anxiety disorders. Importantly, despite this possibility of a reduction in ERS behaviour due to medication use, individuals with OCD in the present study still reported engaging in greater levels of ERS than healthy controls.

Although control participants with anxiety scores that fell greater three standard deviations away from the group mean or that fell in the "severe" range were removed from these analyses, several participants did report moderate levels of anxiety based on the DASS-21. Since ERS is highly linked to anxiety, the possibility that the presence of anxiety symptoms among participants in the control group may have impacted the results was explored (Parrish \& Radomsky, 2010). However, when analyses were conducted excluding participants with moderate DASS-21 anxiety subscale scores, significant differences remained between the level of ERS in response to general threats sought between individuals with OCD and healthy controls.

The findings from hypothesis 1a contribute importantly to the literature, as they provide novel information regarding the situations in which individuals with OCD appear to seek reassurance. Parrish and Radomsky (2011) previously investigated this question using interview methodology and found that individuals with OCD reported engaging in ERS most frequently in response to general threats. The present study expanded upon previous work in this area by using 
a well-validated measure of ERS for anxiety disorder populations and providing a direct comparison between OCD patients and healthy controls (Rector et al., 2011). With these modifications, the findings from the present study corroborate those from the work of Parrish and Radomsky (2011), and also extend this work by demonstrating that individuals with OCD report seeking greater levels of ERS than healthy controls in response to general, decisionrelated, and social threats.

Interestingly, Hypothesis $1 \mathrm{~b}$ was not supported; results indicated that individuals with OCD with and without primary checking compulsions did not differ significantly in the degree to which they reported engaging in ERS in response to general, decision-related, or social threats. This is surprising given that past research has indicated that ERS in response to general threats is highly correlated with checking compulsions on the Y-BOCS (Starcevic et al., 2012). The high co-occurrence of these two symptoms was replicated in the present study, where ERS in response to general threats and checking symptoms co-occurred at a rate of $95.65 \%$. However, for Hypothesis $1 \mathrm{~b}$, the present study more specifically examined whether those who reported checking behaviour as a primary compulsion on the Y-BOCS reported seeking greater reassurance than those who did not report checking as a primary compulsive behaviour. The results from this novel analysis demonstrate that although checking behaviour and ERS frequently co-occur, those who report checking symptoms as a primary compulsion do not appear to seek greater levels of ERS than those with different primary compulsive behaviours.

\section{Cognitive Correlates of ERS}

Past research has identified similarities between checking behaviour and ERS among individuals with OCD. Specifically, both behaviours appear to serve the function of reducing 
anxiety and preventing anticipated harm (Rachman, 2002). Due to these similarities, it was hypothesized that the cognitive deficits associated with checking behaviour among individuals with OCD may also be related to ERS behaviour in response to general threats, the most common form of ERS endorsed by this population (Parrish \& Radomsky, 2010).

Correlational analyses were conducted to determine which areas of cognitive functioning were significantly related to ERS in response to general threats. Contrary to the predicted outcome for Hypothesis 2, none of the cognitive functions examined, including planning, response inhibition, set-shifting, sustained attention, processing speed, encoding, and verbal and non-verbal memory were found to relate significantly to ERS in response to general threats.

Thus, none of the variables that were shown to be significantly related to the checking subtype in the meta-analysis by Leopold and Backenstrass (2015) were associated with ERS in response to general threats in the present study. This differentiates ERS from checking behaviour, and provides novel information that even the cognitive correlates that are most closely linked to OCD checking (i.e., those with large effect sizes such as planning and response inhibition) do not appear to be related to ERS behaviour (Leopold \& Backenstrass, 2015). As previously mentioned, despite the similar functions that ERS and checking behaviour serve, compulsive checking is usually performed when individuals are alone (Rachman, 2002), and ERS is an interpersonal behaviour wherein the individual is seeking information and reassurance from others (Starcevic et al., 2012; Williams et al., 2012). Therefore, given their differing behavioural manifestations, it is perhaps not surprising that these two behaviours would each be associated with different cognitive functions.

It is also important to note that there are differences between the methodology of present study and past research on cognitive impairment and checking behaviour. The present study 
investigated ERS and cognitive functioning within a sample of participants with varying symptom presentations of OCD. In contrast, in the checking behaviour literature, cognitive functioning has generally been compared between individuals with the OCD checking subtype and the OCD contamination/cleaning subtype (Leopold \& Backenstrass, 2015). Therefore, the cognitive correlates identified for checking behaviour thus far are specifically related to the OCD checking subtype. To clarify differences between the cognitive correlates of checking behaviour and those of ERS, researchers should replicate the present study using a larger sample size to examine the relationship between both ERS and checking behaviour, and performance in each of these cognitive domains, across individuals with various symptom presentations of OCD. Using a similar methodology to the present study will allow for direct comparisons between the cognitive correlates associated with each of these two behaviours.

Importantly, the present study was the first to examine the cognitive correlates of ERS behaviour. This study's novel findings suggest that a number of specific cognitive functions including planning, response inhibition, set-shifting, sustained attention, processing speed, encoding, and verbal and non-verbal memory do not appear to be cognitive correlates of ERS in response to general threats.

\section{Reassurance-Seeking and Decision-Making}

The IGT ambiguous decision-making task consists of five sets of trials (Bechara et al., 2007). In the present study, OCD patients showed significant impairment relative to healthy controls on the last set of trials, while performance did not differ significantly between the two groups for the first four sets of trials. These results are similar to findings from past studies in which OCD patients have consistently shown impairment on later trials of the IGT, compared to 
healthy controls (Kim et al., 2015; Rocha, Alvarenga, Malloy-Diniz, \& Corrêa, 2011; Zhang et al., 2015).

In order to perform well on the IGT, participants must learn to choose cards from the decks that are associated with fewer large losses, despite the fact that these same decks do not provide monetary gains as large as those obtained by choosing the riskier decks (Brand et al., 2006). Although performance may be somewhat indistinguishable between OCD patients and healthy controls on the earlier trials of the IGT as participants are learning how to play the game, performance appears to diverge between these two groups as the task progresses. Specifically, healthy controls begin to take in information about which decks are less advantageous and this group appears to develop an explicit strategy for avoiding these decks in order to obtain a higher overall score (Kim et al., 2015). Researchers have suggested that greater impairment on later trials of the IGT may indicate that OCD patients are less able or unable to develop a specific strategy for selecting from the advantageous decks and thus ending up with a greater sum of money (Kim et al., 2015).

Consistent with Hypothesis 3, a significant negative relationship was identified between ERS in response to decision-related threats and overall performance on the IGT as indicated by the total amount of money remaining at the end of the task. Thus, seeking high levels of reassurance in response to decision-related threats was associated with greater impairment on the IGT. Supplementary analyses were conducted to examine the relationship between ERS in response to decision-related threats and OCD patients' IGT performance at various points throughout the IGT task. Net performance on the first set of trials was not significantly related to decision-related ERS. However, on all subsequent trials with the exception of the third set of trials, a significant relationship between decision-making performance and decision-related ERS 
emerged. These findings may be related to the fact that performance on the beginning trials of the IGT do not involve strategy given that all participants are unaware of the losses and gains associated with each deck. Thus, decision-making ability may not be captured until several trials into the task and the relationship between ERS and performance on the first set of trials of the IGT may therefore not be representative of how ambiguous decision-making and ERS are related.

However, overall impairment in performance on the IGT, as well as impairment on later trials of the IGT, were significantly associated with greater decision-related ERS. This relationship may occur as a result of poor decision-making abilities leading OCD patients to doubt themselves, which may therefore cause them to seek reassurance from others in an effort to mitigate this doubt. Past research has suggested that doubt and poor decision-making are linked (Dittrich \& Johansen, 2013). This possibility highlights the importance of OCD patients' perceptions of their decision-making abilities. The relationship between self-reported indecisiveness and ERS in response to decisions was therefore also examined in the present analysis.

In support of hypothesis 4, a significant relationship was found between self-reported indecisiveness on the Frost Indecisiveness Scale and ERS in response to decision-related threats on the RSS among individuals with OCD. Given that responses on the FIS are self-reported and therefore represent participants' perceptions of their ability to make appropriate decisions, it is likely that their reports of indecisiveness on the FIS are not reflective of objective indecisiveness. Interestingly, the effect size for the relationship between decision-related ERS and indecisiveness was stronger than that identified between decision-related ERS and objective ambiguous decision-making performance in the present analysis. This indicates that one's perception of his 
or her decision-making skills, or one's confidence in his or her decision-making abilities, is more closely related to decision-related ERS than actual decision-making performance.

These novel findings demonstrate a possible benefit of targeting decision-making in interventions for OCD patients who exhibit decision-related ERS. Because decision-related ERS and ambiguous decision-making are related, it is possible that improving decision-making skills in psychotherapy would lead to a decrease in decision-related ERS. Strategies for targeting decision-related ERS have not been examined to date in the literature. Furthermore, the literature on strategies for improving decision-making skills has not yet been extended to OCD populations. However, cognitive behavioural therapy and mindfulness strategies appear to be effective for improving decision-making skills in other populations (Karelaia \& Reb, 2014; Oldershaw et al., 2012). For example, decision-making training is often used in cognitive behavioural therapy with compulsive hoarders and has been found to be successful in improving decision-making in this population (Hartl \& Frost, 1999). Given that hoarding disorder and OCD share common features and are often comorbid, these strategies may also prove to be effective for targeting decision-making skills in individuals with OCD who exhibit high levels of decisionrelated ERS (Frost, Krause, \& Steketee, 1996). Importantly, given the strength of the relationship between decision-related ERS and self-reported indecisiveness, it is also possible that increasing one's perception of his or her decision-making abilities without improving actual decisionmaking performance would be sufficient to reduce decision-related ERS. The relationship between cognitive confidence and ERS is discussed next. 


\section{Reassurance-Seeking and Cognitive Confidence}

Cognitive confidence is defined as one's perception of his or her cognitive functioning.

On the MACCS, higher scores indicate greater endorsements of low cognitive confidence (Nedeljkovic \& Kyrios, 2007). Hypothesis 5a from the present study was not supported; the relationship between overall cognitive confidence and ERS in response to general threats was not found to be significant among OCD patients. This lack of relationship may be due to a ceiling effect, given the consistently high levels of ERS in response to general threats reported by this group, as compared to the greater variability in scores of ERS in response to decision-related threats.

In support of Hypotheses $5 \mathrm{~b}$ and 6 , significant negative relationships were identified between decision-related cognitive confidence and ERS in response to decision-related threats, as well as between overall cognitive confidence and ERS in response to decision-related threats among individuals with OCD. Specifically, seeking high levels of reassurance in response to decision-related threats was associated with lower overall cognitive confidence as well as lower confidence in one's decision-making abilities specifically. Similar to the relationships identified in Hypotheses 3 and 4, this finding appears to highlight the role that doubting one's ability to make independent decisions plays in seeking reassurance from others about decisions one must make.

Cognitive confidence also includes one's beliefs about his or her ability to remember information and concentrate, and the standards one has for his or her cognitive performance (Nedeljkovic \& Kyrios, 2007). Thus, the significant relationship identified between decisionrelated ERS and overall cognitive confidence indicates that perhaps doubting one's general cognitive functions also leads individuals with OCD to seek reassurance about decisions out of 
concern that they will not remember an important piece of information involved in making the decision, that they will not be able to focus well enough to make the decision independently, or that they will not live up to their personal standards for making the right decision if they make the decision without seeking input from others. Further research should be done to examine these possible contributing factors, as well as the causal nature of these two relationships. This is the first study to date to examine the link between cognitive confidence and ERS.

The finding that cognitive confidence plays a role in the degree to which someone engages in decision-related ERS may provide key information for treating individuals with OCD who engage in ERS when making decisions. Although future research must elucidate the presence of a causal relationship, targeting decision-making confidence and overall cognitive confidence could lead to reductions in decision-related ERS. Cognitive confidence could be targeted through psychoeducation or through cognitive behavioural strategies such as cognitive restructuring and behavioural experiments. No research to date has been done to investigate the impact of such interventions on ERS behaviour. However, past research has found that maladaptive beliefs about one's cognitive abilities can be targeted through a brief, two session cognitive intervention (Alcolado \& Radomsky, 2016). The intervention includes two main components: psychoeducation about typical beliefs about cognitive functioning in OCD populations, and behavioural experiments in which participants test their beliefs and assumptions about their memory. This brief cognitive intervention also led to decreases in checking behaviour among individuals with OCD (Alcolado \& Radomsky, 2016). Similar interventions may be effective for targeting decision and cognitive confidence, and decision-related ERS. 


\section{Intolerance of Uncertainty and Decision-Making}

Hypothesis 7a was not supported; a significant relationship did not emerge between overall IU scores and IGT performance in this sample. With respect to Hypothesis 7b, a significant negative relationship was found between inhibitory IU as measured by the IUS-12 inhibitory anxiety subscale, and performance on the IGT, as measured by the amount of money participants had remaining at the end of the task. Thus, higher levels of inhibitory IU were related to greater impairment on the IGT. However, no significant relationship was identified between prospective IU scores and IGT performance. Consistent with Hypotheses $8 \mathrm{a}$ and $8 \mathrm{~b}$, a significant positive relationship was identified between total IU scores and self-reported indecisiveness, and between inhibitory IU and indecisiveness, among individuals with OCD. This indicates that greater levels of overall IU and inhibitory IU are associated with greater levels of indecisiveness. No significant relationship was found between prospective IU and selfreported indecisiveness scores.

These findings indicate that high levels of subjective reports of indecisiveness and high levels of impairment on objective ambiguous decision-making tasks are both related to increased inhibitory IU. The present study was the first to find that inhibitory IU, and not prospective IU, is related to decision-making. Whereas inhibitory IU involves uncertainty related to one's ability to inhibit a behaviour or experience, prospective IU is related to fear of uncertainty about future events (Meeten et al., 2012). Thus, prospective IU is more future-oriented than inhibitory IU and therefore the tendency to engage in this form of IU may be less likely to impact decisiveness or performance on a decision-making task. On the other hand, inhibitory IU relates to paralysis in the face of uncertainty, and concern about being able to prevent an incorrect response or decision; therefore, engaging in this form of IU may distract individuals with OCD when they 
are making decisions, thus leading them to become indecisive and to exhibit poor decisionmaking performance.

It is also interesting to note that whereas the relationship between overall IU and ambiguous decision-making performance was not significant, a significant relationship was identified between overall IU and self-reported indecisiveness. It is possible that IU promotes this relationship by leading to concern which becomes distracting and causes these individuals to struggle with making a decision. The discrepancy between these two findings suggests that the overarching IU construct is related to perceptions of indecisiveness, but not to objective ambiguous decision-making performance. This may not be surprising given that effect sizes suggest that the strength of the relationship between self-reported indecisiveness and inhibitory IU is stronger than that of the relationship between ambiguous decision-making performance and inhibitory IU. This finding suggests that among OCD patients, the inability to tolerate ambiguity or uncertainty appears to be particularly importantly linked to perceived indecisiveness.

The novel finding that inhibitory IU is related to ambiguous decision-making performance points to decision-making as a possible area of focus for interventions targeting inhibitory IU specifically. As previously noted, cognitive behavioural therapy strategies can include decision-making training and teaching this skill has been shown to improve decisionmaking skills (Oldershaw et al., 2012). Furthermore, mindfulness strategies appear to be another effective method for improving decision-making (Karelaia \& Reb, 2014). Given that a significant relationship was also identified between self-reported indecisiveness and inhibitory IU, perceptions of one's decision-making abilities may also be an effective target for this behaviour. Specifically, interventions that include psychoeducation and testing behavioural 
assumptions about decision-making abilities may prove beneficial for targeting this behaviour (Alcolado \& Radomsky, 2016).

\section{Limitations}

This study has three main limitations. Firstly, the questionnaire data from this study is limited by its self-report nature. Previous literature suggests that self-report data is open to individual bias, and therefore individuals may tend to either over or under-report symptoms (Van de Mortel, 2008). However, to minimize the risk of this potential bias, the present study provided clear explanations of all questionnaires to participants, and participants were informed that they could ask the study investigators for clarification on any of the questions. Furthermore, the investigators ensured that participants were paying attention throughout the study and there was no evidence of negative test effort among any of the participants. In addition, an objective measure of test effort was obtained during the assessment via the California Verbal Learning Test and results from this measure indicated that all participants in both groups were putting forth sufficient effort.

Secondly, the sample size of the present study did not allow for the relationships that were examined herein to be studied among particular OCD subtypes, such as the OCD checking subtype. However, the sample size was sufficient for detecting these relationships among OCD patients generally. Thirdly, it is important to note that analyses for Hypotheses 2, 3, 4, 5a, 5b, 6, 7a, $7 \mathrm{~b}, 8 \mathrm{a}$, and $8 \mathrm{~b}$ were correlational and thus causality cannot be concluded from the present study's findings. The correlational nature of the analyses also introduces the possibility that other factors may be playing a role in the relationships examined. Nevertheless, and importantly, this 
study was able to point to multiple novel relationships among OCD patients, which will greatly inform future research about these behaviours.

\section{Future Directions}

The relationships identified in the present study provide important groundwork from which subsequent research on ERS and IU in OCD populations can draw. For example, this is the first study to identify ERS in response to social threats as a possible symptom of OCD. Thus, future researchers should continue to investigate differences in the nature of ERS behaviour in varying samples of OCD patients, including those with and without comorbid depression.

Furthermore, given that the present investigation was the first study to examine the cognitive correlates of ERS, further work needs to be done in this area using larger samples before conclusive implications can be drawn. The present investigation should also be extended to examine the cognitive correlates of checking behaviour in a group of OCD patients who exhibit this behaviour but who do not necessarily fit the checking subtype. This will allow for more general conclusions to be drawn about the cognitive correlates associated with checking behaviour in OCD patients and will allow for comparisons to be made between the correlates associated with ERS and those associated with checking behaviour.

This study also provides support for examining the effects of interventions such as cognitive-behavioural therapy, mindfulness, and neurocognitive training in targeting decisionmaking or decision-making confidence to decrease ERS behaviour and IU in OCD populations.

Another possible future direction includes collecting a larger sample size that includes a sufficient number of OCD patients with various subtypes to allow for separate statistical analyses. The relationships between various psychological phenomena identified herein could 
then be investigated among those with various subtypes to determine whether any differences emerge when these groups are examined separately. In addition, these relationships can be examined among healthy individuals in future research.

\section{Conclusions}

The present investigation represents novel work in the areas of cognitive functioning, ERS, cognitive confidence, and IU in OCD research. Specifically, this study showed that individuals with OCD seek significantly greater levels of ERS than healthy controls in response to general threats, decision-related threats, and social threats. In addition, many novel relationships were identified, including: (1) a significant positive relationship between decisionrelated ERS and objective decision-making impairment under ambiguity, (2) a significant positive relationship between decision-related ERS and subjective reports of indecisiveness, (3) a significant negative relationship between overall cognitive confidence and ERS in response to decision-related threats, (4) a significant negative relationship between decision-making confidence and ERS in response to decision-related threats, (5) a significant positive relationship between inhibitory IU and decision-making impairment under ambiguity, (6) a significant positive relationship between inhibitory IU and self-reported indecisiveness, and (7) a significant positive relationship between overall IU and self-reported indecisiveness. These findings will greatly inform our understanding of ERS and IU among individuals with OCD and will contribute importantly to future interventions and research in this area. 


\section{References}

Abramovitch, A., Abramowitz, J. S., \& Mittelman, A. (2013). The neuropsychology of adult obsessive-compulsive disorder: A meta-analysis. Clinical Psychology Review, 33(8), $1163-$ 71.

Abramowitz, J. S., Franklin, M. E., \& Cahill, S. P. (2003). Approaches to common obstacles in the exposure-based treatment of obsessive-compulsive disorder. Cognitive and Behavioral Practice, $10(1), 14-22$.

Alcolado, G. M., \& Radomsky, A. S. (2016). A novel cognitive intervention for compulsive checking: Targeting maladaptive beliefs about memory. Journal of Behavior Therapy and Experimental Psychiatry, 53, 75-83.

American Psychiatric Association (2013). Diagnostic and statistical manual of mental disorders ( $5^{\text {th }}$ ed.). Washington, DC: American Psychiatric Association.

Antony, M. M., Bieling, P. J., Cox, B. J., Enns, M. W., \& Swinson, R. P. (1998). Psychometric properties of the 42-item and 21-item versions of the Depression Anxiety Stress Scales in clinical groups and a community sample. Psychological Assessment, 10(2), 176.

Baddeley, A. (2012). Working memory: Theories, models, and controversies. Annual Review of Psychology, 63, 1-29.

Bais, F. (2012). Intolerance of uncertainty and its effect on future-oriented decision-making (Master's Thesis). Department of Clinical Psychology, University of Amsterdam.

Banca, P., Vestergaard, M. D., Rankov, V., Baek, K., Mitchell, S., Lapa, T., ... Voon, V. (2015). Evidence accumulation in obsessive-compulsive disorder: The role of uncertainty and monetary reward on perceptual decision-making thresholds. Neuropsychopharmacology, 40(5), 1192-1202. 
Bechara, A., Damasio, A. R., Damasio, H., \& Anderson, S. W. (1994). Insensitivity to future consequences following damage to human prefrontal cortex. Cognition, 50, 7-15.

Beck, A. T., \& Steer, R. A. (1990). Manual for the Beck Anxiety Inventory. San Antonio, TX: Psychological Corporation.

Beck, A. T., Steer, R. A., \& Brown, G. K. (1996). Beck Depression Inventory-II. San Antonio, TX: Psychological Corporation.

Beckstein, C. L. (2001). Gender differences in obsessive compulsive disorder symptomatology. Dissertation Abstracts International: The Sciences and Engineering, 2950. Retrieved from http://search.proquest.com/docview/619720062?accountid=14182 \nhttp://sussexprimo.hosted.exlibrisgroup.com/openurl/44SUS_INST/44SUS_SP??url_ver=Z39.882004\&rft_val_fmt=info:ofi/fmt:kev:mtx:dissertation\&genre=dissertations $+\&+$ theses $\&$ sid $=\mathrm{P}$ roQ:PsycINFO\&atitl

Boelen, P. A., \& Carleton, R. N. (2012). Intolerance of uncertainty, hypochondriacal concerns, obsessive-compulsive symptoms, and worry. The Journal of Nervous and Mental Disease, 200(3), 208-213.

Brakoulias, V. (2014). The future of pharmacotherapy for obsessive-compulsive disorder may lie in a better understanding of its heterogeneity. Expert Opinion on Pharmacotherapy, 15(10), $1321-3$.

Brakoulias, V., Starcevic, V., Berle, D., Milicevic, D., Hannan, A., \& Martin, A. (2014). The relationships between obsessive-compulsive symptom dimensions and cognitions in obsessive-compulsive disorder. Psychiatric Quarterly, 85(2), 133-142.

Brand, M., Labudda, K., Kalbe, E., Hilker, R., Emmans, D., Fuchs, G., ... \& Markowitsch, H. J. (2004). Decision-making impairments in patients with Parkinson's disease. Behavioural 
Neurology, 15(3-4), 77-85.

Brand, M., Recknor, E. C., Grabenhorst, F., \& Bechara, A. (2007). Decisions under ambiguity and decisions under risk: Correlations with executive functions and comparisons of two different gambling tasks with implicit and explicit rules. Journal of Clinical and Experimental Neuropsychology, 29(1), 86-99.

Brand, M., Labudda, K., \& Markowitsch, H. (2006). Neuropsychological correlates of decisionmaking in ambiguous and risky situations. Neural Networks, 19(8), 1266-76.

Buelow, M. T., \& Suhr, J. A. (2009). Construct validity of the Iowa gambling task. Neuropsychology Review, 19(1), 102-114.

Buhr, K., \& Dugas, M. J. (2002). The intolerance of uncertainty scale: Psychometric properties of the English version. Behaviour Research and Therapy, 40(8), 931-945.

Carleton, R. N., Norton, M. P. J., \& Asmundson, G. J. (2007). Fearing the unknown: A short version of the Intolerance of Uncertainty Scale. Journal of Anxiety Disorders, 21(1), 105117.

Cavedini, P., Zorzi, C., Piccinni, M., Cavallini, M. C., \& Bellodi, L. (2010). Executive dysfunctions in obsessive-compulsive patients and unaffected relatives: Searching for a new intermediate phenotype. Biological Psychiatry, 67(12), 1178-84.

Chamberlain, S. R., Fineberg, N. A., Blackwell, A. D., Clark, L., Robbins, T. W., \& Sahakian, B. J. (2007). A neuropsychological comparison of obsessive-compulsive disorder and trichotillomania. Neuropsychologia, 45(4), 654-662.

Cougle, J. R., Fitch, K. E., Fincham, F. D., Riccardi, C. J., Keough, M. E., \& Timpano, K. R. (2012). Excessive reassurance seeking and anxiety pathology: Tests of incremental associations and directionality. Journal of Anxiety Disorders, 26(1), 117-25. 
Culbertson, W. C., \& Zillmer, E. A. (1998). The construct validity of the Tower of London DX as a measure of the executive functioning of ADHD children. Assessment, 5(3), 215-226.

Delis, D. C., Freeland, J., Kramer, J. H., \& Kaplan, E. (1988). Integrating clinical assessment with cognitive neuroscience: Construct validation of the California Verbal Learning Test. Journal of consulting and clinical psychology, 56(1), 123.

Delis, D. C., Kramer, J. H., Kaplan, E., \& Thompkins, B. A. O. (1987). CVLT, California Verbal Learning Test: Adult Version: Manual. Psychological Corporation.

Dittrich, W. H., \& Johansen, T. (2013). Cognitive deficits of executive functions and decisionmaking in obsessive-compulsive disorder. Scandinavian Journal of Psychology, 54(5), 393400.

Edwards, J. D., Wadley, V. G., Vance, D. E., Wood, K., Roenker, D. L., \& Ball, K. K. (2005). The impact of speed of processing training on cognitive and everyday performance. Aging \& Mental Health, 9(3), 262-271.

Fals-Stewart, W. (1992). An interrater reliability study of the Trail Making Test (Parts A and B). Perceptual and Motor Skills, 74(1), 39-42.

First, M. B., Williams, J. B. W., Karg, R. S., \& Spitzer, R. L. (2014). Structured Clinical Interview for DSM-5 Disorders-Research Version (SCID-5-RV). Arlington: American Psychiatric Association.

Freeston, M. H., Rhéaume, J., Letarte, H., Dugas, M. J., \& Ladouceur, R. (1994). Why do people worry? Personality and Individual Differences, 17(6), 791-802.

Frost, R. O., Krause, M. S., \& Steketee, G. (1996). Hoarding and obsessive-compulsive symptoms. Behavior Modification, 20(1), 116-132.

Frost, R. O., \& Shows, D. L. (1993). The nature and measurement of compulsive indecisiveness. 
Behaviour Research and Therapy, 31(7).

Gentes, E. L., \& Ruscio, A. M. (2011). A meta-analysis of the relation of intolerance of uncertainty to symptoms of generalized anxiety disorder, major depressive disorder, and obsessive-compulsive disorder. Clinical Psychology Review, 31(6), 923-933.

Goodman, W. K., Price, L. H., Rasmussen, S. A., Mazure, C., Fleischmann, R. L., Hill, C. L., ... \& Charney, D. S. (1989). The Yale-Brown Obsessive Compulsive Scale: I. Development, use, and reliability. Archives of General Psychiatry, 46(11), 1006-1011.

Hartl, T. L., \& Frost, R. O. (1999). Cognitive-behavioral treatment of compulsive hoarding: a multiple baseline experimental case study. Behaviour Research and Therapy, 37(5), 451461.

Hashimoto, N., Nakaaki, S., Omori, I. M., Fujioi, J., Noguchi, Y., Murata, Y., ... \& Furukawa, T. A. (2011). Distinct neuropsychological profiles of three major symptom dimensions in obsessive-compulsive disorder. Psychiatry Research, 187(1), 166-173.

Henry, J. D., \& Crawford, J. R. (2005). The short-form version of the Depression Anxiety Stress Scales (DASS-21): Construct validity and normative data in a large non-clinical sample. British Journal of Clinical Psychology, 44(2), 227-239.

Helsen, K., Van den Bussche, E., Vlaeyen, J. W., \& Goubert, L. (2013). Confirmatory factor analysis of the Dutch Intolerance of Uncertainty Scale: Comparison of the full and short version. Journal of Behavior Therapy and Experimental Psychiatry, 44(1), 21-29.

Hermans, D., Engelen, U., Grouwels, L., Joos, E., Lemmens, J., \& Pieters, G. (2008). Cognitive confidence in obsessive-compulsive disorder: Distrusting perception, attention and memory. Behaviour Research and Therapy, 46(1), 98-113.

Holdnack, H.A. (2001). Wechsler Test of Adult Reading: WTAR. San Antonio: The 
Psychological Corporation.

Honkonen, T., Virtanen, M., Ahola, K., Kivimäki, M., Pirkola, S., Isometsä, E., ... \& Lönnqvist, J. (2007). Employment status, mental disorders and service use in the working age population. Scandinavian Journal of Work, Environment, and Health, 33(1), 29-36.

Jacoby, R. J., Fabricant, L. E., Leonard, R. C., Riemann, B. C., \& Abramowitz, J. S. (2013). Just to be certain: Confirming the factor structure of the Intolerance of Uncertainty Scale in patients with obsessive-compulsive disorder. Journal of Anxiety Disorders, 27(5), 535-542.

Joiner Jr, T. E., \& Metalsky, G. I. (2001). Excessive reassurance seeking: Delineating a risk factor involved in the development of depressive symptoms. Psychological Science, 12(5), 371-378.

Karelaia, N., \& Reb, J. (2014). Improving Decision Making Through Mindfulness. Forthcoming in J. Reb \& P. Atkinds (Eds.), Mindfulness in Organizations. Cambridge University Press.

Katrin Kuelz, A., Riemann, D., Zahn, R., \& Voderholzer, U. (2004). Object alternation test—is it sensitive enough to detect cognitive dysfunction in obsessive-compulsive disorder? European Psychiatry, 19(7), 441-443.

Khawaja, N. G., \& Yu, L. N. H. (2010). A comparison of the 27-item and 12-item intolerance of uncertainty scales. Clinical Psychologist, 14(3), 97-106.

Kessler, R. C., \& Frank, R. G. (1997). The impact of psychiatric disorders on work loss days. Psychological Medicine, 27(04), 861-873.

Kim, H. W., Kang, J. I., Namkoong, K., Jhung, K., Ha, R. Y., \& Kim, S. J. (2015). Further evidence of a dissociation between decision-making under ambiguity and decision-making under risk in obsessive-compulsive disorder. Journal of Affective Disorders, 176, 118-24.

Kobori, O., \& Salkovskis, P. M. (2013). Patterns of reassurance seeking and reassurance-related 
behaviours in OCD and anxiety disorders. Behavioural and Cognitive Psychotherapy, $41(1), 1-23$.

Kolada, J. L., Bland, R. C., \& Newman, S. C. (1994). Obsessive-Compulsive Disorder. Acta Psychiatrica Scandinavica, 89(376), 24-35.

Koran L. M., Thienemann M. L., Davenport R. (1996). Quality of life for patients with obsessive-compulsive disorder. American Journal of Psychiatry, 153, 783-788.

Kortte, K. B., Horner, M. D., \& Windham, W. K. (2002). The trail making test, part B: cognitive flexibility or ability to maintain set?. Applied Neuropsychology, 9(2), 106-109.

Laitinen-Krispijn, S., \& Bijl, R. V. (2000). Mental disorders and employee sickness absence: The NEMESIS study. Social psychiatry and psychiatric epidemiology, 35(2), 71-77.

Lawrence, N. S., Wooderson, S., Mataix-Cols, D., David, R., Speckens, A., \& Phillips, M. L. (2006). Decision making and set shifting impairments are associated with distinct symptom dimensions in obsessive-compulsive disorder. Neuropsychology, 20(4), 409.

Leopold, R., \& Backenstrass, M. (2015). Neuropsychological differences between obsessivecompulsive washers and checkers: A systematic review and meta-analysis. Journal of Anxiety Disorders, 30, 48-58.

Lezak, M. D. (1995). Neuropsychological assessment (3rd ed.). New York: Oxford University Press.

Lochner, C., Seedat, S., du Toit, P. L., Nel, D. G., Niehaus, D. J. H., Sandler, R., \& Stein, D. J. (2005). Obsessive-compulsive disorder and trichotillomania: A phenomenological comparison. BMC Psychiatry, 5, 2.

Logan, G. D., Cowan, W. B., \& Davis, K. A. (1984). On the ability to inhibit simple and choice reaction time responses: A model and a method. Journal of Experimental Psychology: 
Human Perception and Performance, 10(2), 276.

Lovibond, S. H., \& Lovibond, P. F. (1995). Manual for the depression anxiety stress scales (2nd ed.). Sydney: Psychology Foundation.

Luhmann, C. C., Ishida, K., \& Hajcak, G. (2011). Intolerance of uncertainty and decisions about delayed, probabilistic rewards. Behavior Therapy, 42(3), 378-386.

Mangiafico, S.S. (2016). Summary and analysis of extension program evaluation in R, version 1.6.19. Retrieved from rcompanion.org/documents/RHandbookProgramEvaluation.pdf .

McEvoy, P. M., \& Mahoney, A. E. (2011). Achieving certainty about the structure of intolerance of uncertainty in a treatment-seeking sample with anxiety and depression. Journal of Anxiety Disorders, 25(1), 112-122.

Meeten, F., Dash, S. R., Scarlet, A. L. S., \& Davey, G. C. L. (2012). Investigating the effect of intolerance of uncertainty on catastrophic worrying and mood. Behaviour Research and Therapy, 50(11), 690-698.

Menzies, L., Chamberlain, S. R., Laird, A. R., Thelen, S. M., Sahakian, B. J., \& Bullmore, E. T. (2008). Integrating evidence from neuroimaging and neuropsychological studies of obsessive-compulsive disorder: The orbitofronto-striatal model revisited. Neuroscience \& Biobehavioral Reviews, 32(3), 525-549.

Meyers, J. E., \& Meyers, K. R. (1995). Rey Complex Figure Test and recognition trial: RCFT. Psychological Assessment Resources.

Nedeljkovic, M., \& Kyrios, M. (2007). Confidence in memory and other cognitive processes in obsessive-compulsive disorder. Behaviour Research and Therapy, 45(12), 2899-2914.

Nedeljkovic, M., Moulding, R., Kyrios, M., \& Doron, G. (2009). The relationship of cognitive confidence to OCD symptoms. Journal of Anxiety Disorders, 23(4), 463-468. 
Nie, N. H., Bent, D. H., \& Hull, C. H. (1970). SPSS: Statistical Package for the Social Sciences. New York: McGraw-Hill.

Nielen, M. M. A., Veltman, D. J., de Jong, R., Mulder, G., \& den Boer, J. A. (2002). Decision making performance in obsessive compulsive disorder. Journal of Affective Disorders, $69(1-3), 257-260$.

Nouchi, R., Taki, Y., Takeuchi, H., Hashizume, H., Akitsuki, Y., Shigemune, Y., ... \& Kawashima, R. (2012). Brain training game improves executive functions and processing speed in the elderly: A randomized controlled trial. PloS one, 7(1), e29676.

Olley, A., Malhi, G., \& Sachdev, P. (2007). Memory and executive functioning in obsessivecompulsive disorder: A selective review. Journal of Affective Disorders, 104(1-3), 1523.

Osborne, J. W., \& Overbay, A. (2004). The power of outliers (any why researchers should always check for them). Practical Assessment, Research, \& Evaluation, 9(6), 1-12.

Osman, A., Wong, J. L., Bagge, C. L., Freedenthal, S., Gutierrez, P. M., \& Lozano, G. (2012). The depression anxiety stress Scales-21 (DASS-21): Further examination of dimensions, scale reliability, and correlates. Journal of Clinical Psychology, 68(12), $1322-1338$.

Osterrieth, P. A. (1944). Le test de copie d'une figure complexe. Archives de Psychologie, 30, 206-356.

Parrish, C. L. (2009). An investigation of excessive reassurance seeking in OCD. (Doctoral Dissertation). Department of Psychology, Concordia University. Retrieved from http://ezproxy.nottingham.ac.uk/login??url=http://search.proquest.com/docview/607047743 ?accountid=8018\nhttp://sfx.nottingham.ac.uk/sfx_local/?url_ver=Z39.88- 
2004\&rft_val_fmt=info:ofi/fmt:kev:mtx:dissertation\&genre=dissertations $+\&+$ theses\&sid $=P$ roQ:ProQuest

Parrish, C. L., \& Radomsky, A. S. (2010). Why do people seek reassurance and check repeatedly? An investigation of factors involved in compulsive behavior in OCD and depression. Journal of Anxiety Disorders, 24(2), 211-222.

Pushkarskaya, H., Tolin, D., Ruderman, L., Kirshenbaum, A., Kelly, J. M., Pittenger, C., \& Levy, I. (2015). Decision-making under uncertainty in obsessive-compulsive disorder. Journal of Psychiatric Research, 69, 166-173.

Rachman, S. (2002). A cognitive theory of compulsive checking. Behaviour Research and Therapy, 40(6), 625-639.

Radomsky, A. S., Dugas, M. J., Alcolado, G. M., \& Lavoie, S. L. (2014). When more is less: Doubt, repetition, memory, metamemory, and compulsive checking in OCD. Behaviour Research and Therapy, 59, 30-39.

Rasmussen, S. A., Eisen, J. L. (1990). Epidemiology of obsessive-compulsive disorder. Journal of Clinical Psychiatry, 51(2), 10-13.

Rassin, E., Muris, P., Franken, I., Smit, M., \& Wong, M. (2007). Measuring general indecisiveness. Journal of Psychopathology and Behavioral Assessment, 29(1), 61-68.

Rector, N. A., Kamkar, K., Cassin, S. E., Ayearst, L. E., \& Laposa, J. M. (2011). Assessing excessive reassurance seeking in the anxiety disorders. Journal of Anxiety Disorders, 25(7), $911-7$.

Rocha, F. F. D., Alvarenga, N. B., Malloy-Diniz, L., \& Corrêa, H. (2011). Decision-making impairment in obsessive-compulsive disorder as measured by the Iowa Gambling Task. Arquivos de Neuro-psiquiatria, 69(4), 642-647. 
Rosario-Campos, M. C., Miguel, E. C., Quatrano, S., Chacon, P., Ferrao, Y., Findley, D., ... \& Tolin, D. (2006). The Dimensional Yale-Brown Obsessive-Compulsive Scale (DY-BOCS): An instrument for assessing obsessive-compulsive symptom dimensions. Molecular psychiatry, 11(5), 495-504.

Rogers, R. D., Owen, A. M., Middleton, H. C., Williams, E. J., Pickard, J. D. Sahakian, B. J. (1999). Choosing between small, likely rewards and large, unlikely rewards activates inferior and orbital prefrontal cortex. Journal of Neuroscience, 19, 9029-9038.

Ruff, R. M., Niemann, H., Allen, C. C., Farrow, C. E., \& Wylie, T. (1992). The Ruff 2 And 7 selective attention test: A neuropsychological application. Perceptual and Motor Skills, 75(3), 1311-1319.

Salkovskis, P. M. (1999). Understanding and treating obsessive-compulsive disorder. Behaviour Research and Therapy, 37, 29-52.

Samani, S., \& Joukar, B. (2007). A study on the reliability and validity of the short form of the depression anxiety stress scale (DASS-21). Journal of Social Sciences and Humanities of Shiraz University, 26(3), 65-77.

Sanchez-Cubillo, I., Perianez, J. A., Adrover-Roig, D., Rodriguez-Sanchez, J. M., Rios-Lago, M., Tirapu, J. E. E. A., \& Barcelo, F. (2009). Construct validity of the Trail Making Test: role of task-switching, working memory, inhibition/interference control, and visuomotor abilities. Journal of the International Neuropsychological Society, 15(3), 438.

Sandson, J., \& Albert, M. L. (1984). Varieties of perseveration. Neuropsychologia, 22(6), 715732.

Schachar, R. J., Chen, S., Logan, G. D., Ornstein, T. J., Crosbie, J., Ickowicz, A., \& Pakulak, A. (2004). Evidence for an error monitoring deficit in attention deficit hyperactivity 
disorder. Journal of Abnormal Child Psychology, 32(3), 285-293.

Schlotzhauer, S. D. (2007). Elementary statistics using JMP. SAS Institute.

Shallice, T. (1982). Specific impairments of planning. Philosophical Transactions of the Royal Society of London Series B: Biological Sciences, 298(1089), 199-209.

Shean, G., \& Baldwin, G. (2008). Sensitivity and specificity of depression questionnaires in a college-age sample. The Journal of Genetic Psychology, 169(3), 281-292.

Sheehan, D. V. (2014). The Mini-International Neuropsychiatric Interview, Version 7.0 for DSM-5 (M.I.N.I. 7.0). Medical Outcomes Systems Inc.

Sheehan, D. V., \& Lecrubier, Y. (2010). The Mini International Neuropsychiatric Interview Version 6.0 (MINI 6.0). Jacksonville, FL: Medical Outcomes Systems Inc.

Sheehan, D. V., Lecrubier, Y., Sheehan, K. H., Amorim, P., Janavs, J., Weiller, E., ... \& Dunbar, G. C. (1998). The Mini-International Neuropsychiatric Interview (MINI): The development and validation of a structured diagnostic psychiatric interview for DSM-IV and ICD10. Journal of Clinical Psychiatry.

Sheehan, D. V., Lecrubier, Y., Sheehan, K. H., Janavs, J., Weiller, E., Keskiner, A., ... \& Dunbar, G. C. (1997). The validity of the Mini International Neuropsychiatric Interview (MINI) according to the SCID-P and its reliability. European Psychiatry, 12(5), 232-241.

Sheridan, L. K., Fitzgerald, H. E., Adams, K. M., Nigg, J. T., Martel, M. M., Puttler, L. I., ... \& Zucker, R. A. (2006). Normative Symbol Digit Modalities Test performance in a community-based sample. Archives of Clinical Neuropsychology, 21(1), 23-28.

Shin, N. Y., Lee, T. Y., Kim, E., \& Kwon, J. S. (2013). Cognitive functioning in obsessivecompulsive disorder: A meta-analysis. Psychological Medicine, 44(06), 1121-1130.

Smith A. (1982). The Symbol Digit Modalities Test (SDMT) Symbol Digit Modalities Test: 
manual. Western Psychological Services.

Starcevic, V., Berle, D., Brakoulias, V., Sammut, P., Moses, K., Milicevic, D., \& Hannan, A. (2012). Interpersonal reassurance seeking in Obsessive-compulsive disorder and its relationship with checking compulsions, Psychiatry Research, 200, 560-567.

Starcke, K., Tuschen-Caffier, B., Markowitsch, H., \& Brand, M. (2009). Skin conductance responses during decisions in ambiguous and risky situations in obsessive-compulsive disorder, Cognitive Neuropsychiatry, 14(3), 199-216.

Starcke, K., Tuschen-Caffier, B., Markowitsch, H. J., \& Brand, M. (2010). Dissociation of decisions in ambiguous and risky situations in obsessive-compulsive disorder. Psychiatry Research, 175(3), 114-120.

Subica, A. M., Fowler, J. C., Elhai, J. D., Frueh, B. C., Sharp, C., Kelly, E. L., \& Allen, J. G. (2014). Factor structure and diagnostic validity of the Beck Depression Inventory-II with adult clinical inpatients: Comparison to a gold-standard diagnostic interview. Psychological assessment, 26(4), 1106.

Starr, L. R., \& Davila, J. (2008). Excessive reassurance seeking, depression, and interpersonal rejection: A meta-analytic review. Journal of Abnormal Psychology, 117 (4), 762-775.

Takeuchi, H., Taki, Y., Hashizume, H., Sassa, Y., Nagase, T., Nouchi, R., \& Kawashima, R. (2011). Effects of training of processing speed on neural systems. Journal of Neuroscience, 31(34), 12139-12148.

Thordarson, D. S., Radomsky, A. S., Rachman, S., Shafran, R., Sawchuk, C. N., \& Ralph Hakstian, A. (2004). The Vancouver Obsessional Compulsive Inventory (VOCI). Behaviour Research and Therapy, 42(11), 1289-1314.

Toffolo, M. B. (2015). Better super safe than slightly sorry? Reciprocal relationships between 
checking behavior and cognitive symptoms in obsessive-compulsive disorder. (Doctoral Dissertation). Department of Psychology, Utrecht University.

Tükel, R., Gürvit, H., Ertekin, B. A., Oflaz, S., Ertekin, E., Baran, B., .. Atalay, F. (2012). Neuropsychological function in obsessive-compulsive disorder. Comprehensive Psychiatry, 53(2), $167-75$.

Van de Mortel, T. F. (2008). Faking it: social desirability response bias in self-report research. Australian Journal of Advanced Nursing, 25(4), 40.

Verbruggen, F., \& Logan, G. D. (2008). Response inhibition in the stop-signal paradigm. Trends in Cognitive Sciences, 12(11), 418-424.

Wang, Y. P., \& Gorenstein, C. (2013). Psychometric properties of the Beck Depression Inventory-II: A comprehensive review. Revista Brasileira de Psiquiatria, 35(4), 416-431.

Williams, M., Farris, S., Turkheimer, E., Pinto, A., Ozanick, K., Franklin, M., ... Foa, E. (2012). The myth of the pure obsessional type in obsessive-compulsive disorder. Depression and Anxiety, 28(6), 495-500.

Wingen, M., Bothmer, J., Langer, S., \& Ramaekers, J. G. (2005). Actual driving performance and motor function in health subjects after acute and subchronic treatment with escitalopram, mirtazapine, and placebo: a crossover trial. The Journal of Clinical Psychiatry, 66(4), 436-443.

Woods, S. P., Delis, D. C., Scott, J. C., Kramer, J. H., \& Holdnack, J. A. (2006). The California Verbal Learning Test-second edition: test-retest reliability, practice effects, and reliable change indices for the standard and alternate forms. Archives of Clinical Neuropsychology, 21(5), 413-420.

Woody, S. R., Steketee, G., \& Chambless, D. L. (1995). Reliability and validity of the Yale- 
Brown Obsessive-Compulsive Scale. Behaviour Research and Therapy, 33(5), 597-605.

World Health Organization (1999). The "newly defined"' burden of mental problems. Fact Sheets no 217. WHO, Geneva.

Zhang, L., Dong, Y., Ji, Y., Zhu, C., Yu, F., Ma, H., ... Wang, K. (2015). Dissociation of decision making under ambiguity and decision making under risk: A neurocognitive endophenotype candidate for obsessive-compulsive disorder. Progress in NeuroPsychopharmacology \& Biological Psychiatry, 57, 60-8. 